\title{
CATANESE-CILIBERTO SURFACES OF FIBER GENUS THREE WITH UNIQUE SINGULAR FIBER
}

\author{
HIROTAKA ISHIDA
}

(Received December 17, 2003, revised April 27, 2005)

\begin{abstract}
In this paper, we study a minimal surface of general type with $p_{g}=q=1$, $K_{S}^{2}=3$ which we call a Catanese-Ciliberto surface. The Albanese map of this surface gives a fibration of curves over an elliptic curve. For an arbitrary elliptic curve $E$, we obtain the Catanese-Ciliberto surface which satisfies $\operatorname{Alb}(S) \cong E$, has no $(-2)$-curves and has a unique singular fiber. Furthermore, we show that the number of the isomorphism classes satisfying these conditions is four if $E$ has no automorphism of complex multiplication type.
\end{abstract}

0. Introduction. Let $S$ be a minimal algebraic surface of general type over $\boldsymbol{C}$. A proper surjective morphism $f: S \rightarrow C$ from an algebraic surface $S$ to a non-singular algebraic curve $C$ is called a fibration of curves of genus $g$ if fibers of $f$ are connected and the genus of the generic fiber is $g$. It is important to study the structures of the fibrations for surfaces of general type. For instance, Horikawa studied surfaces with fibrations of curves of genus two $[5,6]$.

We set $p_{g}(S)=\operatorname{dim} H^{2}\left(S, \mathcal{O}_{S}\right)$ and $q(S)=\operatorname{dim} H^{1}\left(S, \mathcal{O}_{S}\right)$. Let $K_{S}^{2}$ be the self intersection number of the canonical divisor $K_{S}$ of $S$. In this paper, we are interested in the case $p_{g}(S)=q(S)=1$ and $K_{S}^{2}=3$. If $q(S)=1$, then the Albanese map $a: S \rightarrow \operatorname{Alb}(S)$ gives a fibration of curves over the elliptic curve $E=\operatorname{Alb}(S)$. Let $g$ be the genus of a general fiber of $a$. Catanese and Ciliberto studied this surface in [2,3] and showed that the genus $g$ is two or three.

Definition. Let $S$ be a minimal algebraic surface of general type over $\boldsymbol{C}$. $S$ is called a Catanese-Ciliberto surface if $S$ satisfies $p_{g}=q=1$ and $K_{S}^{2}=3$.

We also denote by $K_{S}$ the invertible sheaf associated to the divisor $K_{S}$. In the case $g=3$, Catanese and Ciliberto showed that the direct image $V=a_{*} K_{S / E}$ of the relative canonical sheaf $K_{S / E}=K_{S} \otimes_{\mathcal{O}_{S}}\left(a^{*} \Omega_{E}^{1}\right)^{\vee} \cong K_{S}$ is an indecomposable vector bundle of rank three and degree one over the elliptic curve $E$. Therefore, there exists a point $P \in E$ such that det $V \cong \mathcal{O}_{E}(P)$. Let $p: \boldsymbol{P}_{E}\left(a_{*} K_{S}\right) \rightarrow E$ be the $\boldsymbol{P}^{2}$-bundle associated with $a_{*} K_{S}$ and $\omega: S \rightarrow \boldsymbol{P}_{E}\left(a_{*} K_{S}\right)$ the relative canonical map. They obtained the following theorem.

ThEOREM 0.1 (Catanese-Ciliberto [3, Theorem 3.1]). Let S, $a, E, g, P, p$ and $\omega$ be as above, and $H$ the tautological divisor of $\boldsymbol{P}_{E}\left(a_{*} K_{S}\right)$, i.e., it satisfies $p_{*} \mathcal{O}_{\boldsymbol{P}_{E}\left(a_{*} K_{S}\right)}(H) \cong$ $a_{*} K_{S}$. If $g=3$, then we have:

2000 Mathematics Subject Classification. Primary 14D05; Secondary 14J29, 14 D06.

Key words and phrases. Surface of gneral type, fibration of curves, elliptic curve. 
(i) the relative canonical map $\omega$ is a morphism;

(ii) $\omega(S)$ is isomorphic to the canonical model of $S$; and

(iii) $\omega(S)$ is a member of the complete linear system $\left|4 H-p^{*} P\right|$, where $p^{*} P$ is the divisor $p^{-1} P$.

Let $V$ be an indecomposable vector bundle of rank three and degree one over an elliptic curve $E$. The $\boldsymbol{P}^{2}$-bundle $\boldsymbol{P}_{E}(V)$ is uniquely determined up to an isomorphism, since $V$ is uniquely determined up to tensor product with a line bundle of degree 0 by Atiyah [1]. We set $\boldsymbol{P}_{E}=\boldsymbol{P}_{E}(V)$. Let $H$ be the tautological divisor of $\boldsymbol{P}_{E}$. When $(E, V)$ is equal to $\left(E, \alpha_{*} K_{S}\right)$ in Theorem 0.1, the image $\omega(S)$ of $S$ is a relative quartic hypersurface in $\boldsymbol{P}_{E}$ which has at worst rational double points as singularities by Theorem 0.1 (ii). Conversely, it is easy to verify that the minimal model $S$ of a member $S^{\prime} \in\left|4 H-p^{*} P\right|$ which has at worst rational double points is a Catanese-Ciliberto surface with $g=3$.

For an algebraic variety $Z$, we denote by $\chi_{\text {top }}(Z)$ the Euler number of $Z$. Let $T$ be a surface with a fibration $f: T \rightarrow C$ of curves of genus $g$ over a curve $C$ and with at worst rational double points. We set $T_{P}=f^{-1}(P)$ for $P \in C$. The Euler number of the nonsingular model $T^{*}$ of $T$ is given by

$$
\chi_{\text {top }}\left(T^{*}\right)=(2-2 g) \chi_{\text {top }}(C)+\sum_{P \in C}\left(\chi_{\text {top }}\left(T_{P}\right)+2 g-2\right)+\sum_{Q \in \operatorname{Sing} T} r(Q),
$$

where $r(Q)$ is the number of exceptional irreducible curves of a singular point $Q$ of $T$.

Let $S^{\prime} \in\left|4 H-p^{*} P\right|$ be a surface which has at worst rational double points. We apply the equality (1) to the surface $S^{\prime}$ with the fibration $\left.p\right|_{S^{\prime}}: S^{\prime} \rightarrow E$ of curves over the elliptic curve $E$. Let $S^{*}$ be the minimal model of $S^{\prime}$ and $S_{P}^{\prime}$ the fiber of $\left.p\right|_{S^{\prime}}$ at $P \in E$. Since $g=3, \chi_{\text {top }}(E)=0$ and $\chi_{\text {top }}\left(S^{*}\right)=9$, we obtain

$$
\sum_{P \in E}\left(\chi_{\text {top }}\left(S_{P}^{\prime}\right)+4\right)+\sum_{Q \in S^{\prime}} r(Q)=9
$$

by the equality (1). Note that $\chi_{\text {top }}\left(S_{P}^{\prime}\right)+4$ is non-negative and is zero for a non-singular fiber. We call $\chi_{\text {top }}\left(S_{P}^{\prime}\right)+4$ the Euler contribution of a singular fiber $S_{P}^{\prime}$ and $r(Q)$ the Euler contribution of a rational double point $Q$.

Because $S^{\prime}$ has a fibration over a non-singular curve, every singular point of $S^{\prime}$ is contained in a singular fiber of $S^{\prime}$. Therefore, the equality (2) implies that $S^{\prime}$ has at least one singular fiber of $S^{\prime}$ and the number of singular fibers is less than or equal to nine.

It seems that, for a general Catanese-Ciliberto surface, singular fibers of $a$ have one node. In this case, this fibraton has nine singular fibers. Conversely, Catanese-Ciliberto surfaces with a unique singular fiber are most special. We know by the argument of monodromies that, if a surface with a fibration of curves over $\boldsymbol{P}^{1}$ has a singular fiber, then it has another singular fiber. On the other hand, there may exist a surface over an elliptic curve with a unique singular fiber.

DEFINITION. Let $S$ be a Catanese-Ciliberto surface. If $S$ has a unique singular fiber and satisfies $g=3$ and $S \cong \omega(S)$, we call it a Catanese-Ciliberto surface of type I. 
In this paper, we show the following theorem.

THEOREM 0.2. For an arbitrary elliptic curve E, there exists a Catanese-Ciliberto surface $S$ of type I which satisfies $\operatorname{Alb}(S) \cong E$. If E has an automorphism of complex multiplication type, then there exist exactly two isomorphism classes of such surfaces. Otherwise, they have exactly four isomorphism classes.

We show the existence of non-singular surfaces $S^{\prime} \in\left|4 H-p^{*} P\right|$ with a unique singular fiber by giving the defining equations in the $\boldsymbol{P}^{2}$-bundle $\boldsymbol{P}_{E}$. Since $V$ is indecomposable, we cannot take a global homogeneous coordinate system on $\boldsymbol{P}_{E}$. In order to describe the defining equation, we employ the following method which was used by Takahashi [9].

Let $\varphi$ be an isogeny of degree three from an elliptic curve $\tilde{E}$ to $E$. According to Atiyah [1] and Oda [8], the inverse image $\varphi^{*} V$ of $V$ by $\varphi$ decomposes into the direct sum $L_{1} \oplus L_{2} \oplus L_{3}$ of three line bundles $L_{i}(i=1,2,3)$. Then we can take the natural unramified morphism $\Phi: \boldsymbol{P}_{\tilde{E}}\left(\varphi^{*} V\right) \rightarrow \boldsymbol{P}_{E}$ of degree three induced by $\varphi$. In order to describe a minimal canonical surface $T \subset \boldsymbol{P}_{E}$ with $c_{1}^{2}(T)=3 p_{g}(T)$ and $q(T)=1$, Takahashi obtained the defining equation of $\Phi^{-1}(T) \subset \boldsymbol{P}_{\tilde{E}}\left(\varphi^{*} V\right)=\boldsymbol{P}_{\tilde{E}}\left(L_{1} \oplus L_{2} \oplus L_{3}\right)$ which is invariant under the action of $\boldsymbol{Z} / 3 \boldsymbol{Z}$. We apply this method to the Catanese-Ciliberto surfaces with $g=3$. The inverse image $\Phi^{-1}\left(S^{\prime}\right)$ is a relative quartic hypersurface in the $\boldsymbol{P}^{2}$-bundle associated with a direct sum of three line bundles over an elliptic curve. Hence, it is much easier to describe the defining equation of a subvariety in $\boldsymbol{P}_{\tilde{E}}\left(\varphi^{*} V\right)$. In Section 1, we explain Takahashi's method and give an explicit general form of the defining equation of the surface $\Phi^{-1}\left(S^{\prime}\right)$. In Section 2 , we show the existence of non-singular surfaces $S^{\prime} \in\left|4 H-p^{*} P\right|$ with a unique singular fiber by using the defining equations obtained in Section 1. In Section 3, we consider all non-singular surfaces $S^{\prime} \in\left|4 H-p^{*} P\right|$ with a unique singular fiber. In Section 4 , we consider the isomorphism classes of these surfaces. In order to find the number of isomorphism classes of these surfaces, we give the defining equations of these in $\boldsymbol{P}_{E}$ instead of doing it in $\boldsymbol{P}_{\tilde{E}}$.

By using Proposition 2.8, Lemmas 3.4, 4.7, 4.8 and 4.9, we complete the proof of Theorem 0.2 .

1. The defining equation in the $\boldsymbol{P}^{2}$-bundle. Let $E$ be an elliptic curve with the zero element $0_{E}$. It is well-known that there exists an indecomposable vector bundle of rank three and degree one over $E$ (see [1]). We fix an indecomposable vector bundle $V$ of rank three and degree one over $E$ with det $V \cong \mathcal{O}_{E}\left(0_{E}\right)$. Let $p: \boldsymbol{P}_{E}=\boldsymbol{P}_{E}(V)=\operatorname{Proj}\left(\bigoplus_{m} \operatorname{Sym}^{m} V\right) \rightarrow E$ be the $\boldsymbol{P}^{2}$-bundle associated with $V$ over $E$. We denote by $H$ the tautological divisor with $p_{*} \mathcal{O}_{\boldsymbol{P}_{E}}(H) \cong V$. We are going to consider the Catanese-Ciliberto surfaces with $g=3$, which has the image $S^{\prime}$ in $\boldsymbol{P}_{E}$.

For any point $P \in E$, we define the automorphism $T_{P}: E \rightarrow E$ by $T_{P}(Q)=Q+P$ and call $T_{P}$ the translation of $E$ by $P . \boldsymbol{P}_{E}$ is isomorphic to the 3 -fold symmetric product $E^{(3)}$ which is the quotient of $E^{3}$ by the natural action of the symmetric group $S_{3}$ (cf. [1, p. 451]). An automorphism $h$ of $E$ induces the automorphism $h^{(3)}$ of $E^{(3)}$ defined by 
$h^{(3)}\left(P_{1}, P_{2}, P_{3}\right)=\left(h\left(P_{1}\right), h\left(P_{2}\right), h\left(P_{3}\right)\right)$. So a translation $T_{P}$ of $E$ induces an automorphism of the set of linearly equivalent classes which are algebraically equivalent to $m H+n F$ ( $m, n \in \boldsymbol{Z}$ ). Using the following proposition, we see that there exists one-to-one correspondence between the isomorphism classes of Catanese-Ciliberto surfaces with $g=3$ and the isomorphism classes of surfaces in the complete linear system $\left|4 H-p^{*} 0_{E}\right|$ with at worst rational double points.

Proposition 1.1 (Catanese-Ciliberto [3, Proposition 1.5]). Let E, $\boldsymbol{P}_{E}, p, H$ be as above and $F$ an algebraically equivalence class of a fiber of $p$. Then the group of translations of $E$ acts transitively on the set of all linearly equivalent classes which are algebraically equivalent to a divisor in $\boldsymbol{P}_{E}$ if the divisor is not a multiple of $3 H-F$.

In order to obtain the defining equation of $S^{\prime} \in\left|4 H-p^{*} 0_{E}\right|$, we employ the result of Oda [8] as Takahashi used it in [9].

1.1. The isogeny of an elliptic curve with degree three. We first recall the following theorem.

THEOREM 1.2 ([1, 8], [9, Theorem 2.4]). For integers $r, d$, let $\mathcal{E}_{E}(r, d)$ be the set of isomorphism classes of indecomposable vector bundles of rank $r$ and degree $d$ over $E$. Let $\varphi: \tilde{E} \rightarrow E$ be an isogeny of degree $r$.

If $\operatorname{gcd}(r, d)=1$, then the map

$$
\begin{array}{r}
\{L \in \operatorname{Pic}(\tilde{E}) \mid \operatorname{deg} L=d\} \longrightarrow \mathcal{E}_{E}(r, d) \\
\Psi \\
\Psi \\
L \longmapsto
\end{array}
$$

is surjective. Denote $G=\operatorname{ker} \varphi$. Then we obtain

$$
\varphi^{*} \varphi_{*} L \cong \bigoplus_{\sigma \in G} T_{\sigma}^{*} L
$$

Let $\tilde{E}$ be an elliptic curve with zero element $0_{\tilde{E}}$ and $\varphi: \tilde{E} \rightarrow E$ an isogeny of degree three. Applying Theorem 1.2 to the case where $r=3$ and $d=1, V=\varphi_{*} \mathcal{O}_{\tilde{E}}\left(0_{\tilde{E}}\right)$ is an indecomposable bundle of rank three and degree one. Since we have $\varphi_{*} \mathcal{O}_{\tilde{E}}=\mathcal{O}_{E} \oplus M \oplus M^{\otimes 2}$ for a line bundle $M$ such that $M^{\otimes 3} \cong \mathcal{O}_{E}$, we have $\operatorname{det} \varphi_{*} \mathcal{O}_{\tilde{E}} \cong \mathcal{O}_{E}$. Furthermore, since $\operatorname{det} \varphi_{*} \mathcal{O}_{\tilde{E}}\left(0_{\tilde{E}}\right) \cong \operatorname{det} \varphi_{*} \mathcal{O}_{\tilde{E}} \otimes_{\mathcal{O}_{E}} \mathcal{O}_{E}\left(\varphi\left(0_{\tilde{E}}\right)\right)$, we have $\operatorname{det} \varphi_{*} \mathcal{O}_{\tilde{E}}\left(0_{\tilde{E}}\right) \cong \mathcal{O}_{E}\left(0_{E}\right)$.

We set $o=0_{\tilde{E}}$. Let $o^{\prime}$ be a point in $\tilde{E}$ of order three. Denote by $o^{\prime \prime}$ the sum of $o^{\prime}$ and $o^{\prime}$ with respect to the group law of $\tilde{E}$. For any $Q \in \tilde{E}$, we denote by $Q^{\prime}$ and $Q^{\prime \prime}$ the points $T_{o^{\prime}}(Q)$ and $T_{o^{\prime \prime}}(Q)$, respectively. We denote by $[o]$ the divisor $o+o^{\prime}+o^{\prime \prime}$ of degree three on $\tilde{E}$.

If we set $G=\operatorname{ker} \varphi=\left\{o, o^{\prime}, o^{\prime \prime}\right\}$, then we know

$$
\varphi^{*} V \cong \mathcal{O}_{\tilde{E}}(o) \oplus \mathcal{O}_{\tilde{E}}\left(o^{\prime}\right) \oplus \mathcal{O}_{\tilde{E}}\left(o^{\prime \prime}\right)
$$


We set $\tilde{V}=\varphi^{*} V$. Let $\tilde{p}: \boldsymbol{P}_{\tilde{E}}=\boldsymbol{P}_{\tilde{E}}(\tilde{V}) \rightarrow \tilde{E}$ and $p: \boldsymbol{P}_{E}(V) \rightarrow E$ be the $\boldsymbol{P}^{2}$ bundles associated with $\tilde{V}$ and $V$, respectively. Let $\tilde{H}$ be the tautological divisor of $\boldsymbol{P}_{\tilde{E}}$ with $\tilde{p}_{*} \mathcal{O}(\tilde{H}) \cong \tilde{V}$.

Let $Y$ be an algebraic variety and $g: Y \rightarrow E$ a morphism. By [4, II, Proposition 7.12], giving a morphism $f: Y \rightarrow \boldsymbol{P}_{E}$ such that $g=p \circ f$ is equivalent to giving a line bundle $L$ over $Y$ and a surjective map of sheaves on $Y, g^{*} V \rightarrow L$. The surjective map corresponding to $f$ is given by pulling back the natural surjective map $p^{*} V \rightarrow \mathcal{O}_{P_{E}}(H)$.

Since there exists the natural surjective map $\tilde{p}^{*} \varphi^{*} V \cong \tilde{p}^{*} \tilde{V} \rightarrow \mathcal{O}_{\boldsymbol{P}_{\tilde{E}}}(\tilde{H})$, the isogeny $\varphi$ induces the unramified morphism $\Phi: \boldsymbol{P}_{\tilde{E}} \rightarrow \boldsymbol{P}_{E}$ of degree three. Consider the following commutative diagram:

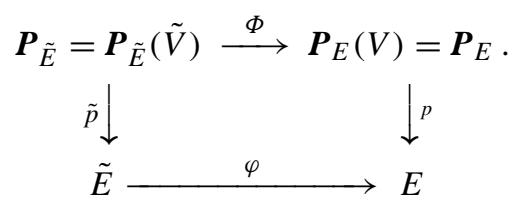

Then we have

$$
\Phi^{*} \mathcal{O}_{\boldsymbol{P}_{E}}\left(4 H-p^{*} 0_{E}\right) \cong \mathcal{O}_{\boldsymbol{P}_{\tilde{E}}}\left(4 \tilde{H}-\tilde{p}^{*}[o]\right) .
$$

The defining polynomial of the inverse image of a surface $S^{\prime} \in\left|4 H-p^{*} 0_{E}\right|$ by $\Phi$ is the element of $H^{0}\left(\boldsymbol{P}_{\tilde{E}}, \mathcal{O}_{\boldsymbol{P}_{\tilde{E}}}\left(4 \tilde{H}-\tilde{p}^{*}[o]\right)\right)$. However, not every member of $H^{0}\left(\boldsymbol{P}_{\tilde{E}}, \mathcal{O}_{\boldsymbol{P}_{\tilde{E}}}(4 \tilde{H}-\right.$ $\left.\left.\tilde{p}^{*}[o]\right)\right)$ gives the inverse image $\Phi^{*} S^{\prime}$ of $S^{\prime}$ in $\left|4 H-p^{*} 0_{E}\right|$. The elements $o, o^{\prime}, o^{\prime \prime}$ of $G$ operate on $H^{0}\left(\boldsymbol{P}_{\tilde{E}}, \mathcal{O}_{\boldsymbol{P}_{\tilde{E}}}\left(4 \tilde{H}-\tilde{p}^{*}[o]\right)\right)$ as $\operatorname{id}_{\boldsymbol{P}_{\tilde{E}}}, T_{o^{\prime}}^{*}, T_{o^{\prime \prime}}^{*}$. Let $H^{0}\left(\boldsymbol{P}_{\tilde{E}}, \mathcal{O}_{\boldsymbol{P}_{\tilde{E}}}\left(4 \tilde{H}-\tilde{p}^{*}[o]\right)\right)^{G}$ be $G-$ invariant subspace of $H^{0}\left(\boldsymbol{P}_{\tilde{E}}, \mathcal{O}_{\boldsymbol{P}_{\tilde{E}}}\left(4 \tilde{H}-\tilde{p}^{*}[o]\right)\right)$. Set $\mathcal{U}=\left\{\operatorname{div}(\Psi) \mid \Psi \in H^{0}\left(\boldsymbol{P}_{\tilde{E}}, \mathcal{O}_{\boldsymbol{P}_{\tilde{E}}}(4 \tilde{H}-\right.\right.$ $\left.\left.\left.\tilde{p}^{*}[o]\right)\right)^{G} \backslash\{0\}\right\}$. Takahashi [9] showed the following.

LEMmA 1.3 (Takahashi [9, Lemma 3.23]). In the above notation, we have

$$
\mathcal{U}=\Phi^{*}\left|4 H-p^{*} 0_{E}\right| .
$$

This lemma implies that $\Phi^{*} \omega(S) \in \mathcal{U}$ for an arbitrary Catanese-Ciliberto surface $S$ with $g=3$. Let $\tilde{S}$ be a member of $\mathcal{U}$ with at worst rational double points. Since $\Psi$ is étale, $\tilde{S} / G \subset \boldsymbol{P}_{E}$ has at worst rational double points. Furthermore, it is easy to see that the minimal model $S$ of the surface $\tilde{S} / G$ is the Catanese-Ciliberto surface with $g=3$ and $\operatorname{Alb}(S) \cong E$. So there exist one-to-one correspondences between isomorphism classes of surfaces with at worst rational double points in the complete linear system $\left|4 H-p^{*} 0_{E}\right|$ and such surfaces in $\mathcal{U} \subset\left|4 \tilde{H}-\tilde{p}^{*}[o]\right|$.

1.2. Defining equation of $\Phi^{*} S^{\prime}$. Let $\boldsymbol{P}_{\tilde{E}}, \tilde{H}, \tilde{p}, o, o^{\prime}, o^{\prime \prime}, G, \mathcal{U}$ be as in Section 1.1. As we saw in Section 1.1, Catanese-Ciliberto surfaces with $g=3$ correspond to surfaces with at worst rational double points in $\mathcal{U}$. We describe explicitly the elements of $H^{0}\left(\boldsymbol{P}_{\tilde{E}}, \mathcal{O}_{P_{\tilde{E}}}(4 \tilde{H}-\right.$ $\left.\left.\tilde{p}^{*}[o]\right)\right)^{G}$ which define surfaces in $\mathcal{U}$. Let $(X: Y: Z)$ be a homogeneous coordinate system of $\boldsymbol{P}^{2}$. We embed the elliptic curve $\tilde{E}$ in $\boldsymbol{P}^{2}$ such that it satisfies the equality $Y^{2} Z=X(X-$ $Z)(X-\lambda Z)$ for $\lambda$ in $\boldsymbol{C} \backslash\{0,1\}$ and $o=(0: 1: 0)$. Let $(\alpha: \beta: 1)$ be the coordinate of $o^{\prime}$. 
Since the order of $o^{\prime}$ is three, we have $o^{\prime \prime}=(\alpha:-\beta: 1), \beta \neq 0$ and the equality

$$
3 \alpha^{4}-4(\lambda+1) \alpha^{3}+6 \alpha^{2} \lambda-\lambda^{2}=0 .
$$

Since $\tilde{p}_{*} \mathcal{O}_{\boldsymbol{P}_{\tilde{E}}}(\tilde{H})=\mathcal{O}_{\tilde{E}}(o) \oplus \mathcal{O}_{\tilde{E}}\left(o^{\prime}\right) \oplus \mathcal{O}_{\tilde{E}}\left(o^{\prime \prime}\right)$, we have $\operatorname{dim} H^{0}\left(\boldsymbol{P}_{\tilde{E}}, \mathcal{O}_{\boldsymbol{P}_{\tilde{E}}}(\tilde{H})\right)=3$. Let $Z_{0}, Z_{1}$ and $Z_{2}$ be the elements of $H^{0}\left(\boldsymbol{P}_{\tilde{E}}, \mathcal{O}_{\boldsymbol{P}_{\tilde{E}}}(\tilde{H})\right)=H^{0}\left(\tilde{E}, \mathcal{O}_{\tilde{E}}(o) \oplus \mathcal{O}_{\tilde{E}}\left(o^{\prime}\right) \oplus \mathcal{O}_{\tilde{E}}\left(o^{\prime \prime}\right)\right)$ which correspond to 1 of $H^{0}\left(\tilde{E}, \mathcal{O}_{\tilde{E}}(o)\right), H^{0}\left(\tilde{E}, \mathcal{O}_{\tilde{E}}\left(o^{\prime}\right)\right)$ and $H^{0}\left(\tilde{E}, \mathcal{O}_{\tilde{E}}\left(o^{\prime \prime}\right)\right)$, respectively. Then we have $Z_{1}=T_{o^{\prime \prime}}^{*} Z_{0}$ and $Z_{2}=T_{o^{\prime}}^{*} Z_{0}$. We set a complex number $\mu$ by $\mu=3 \alpha^{2}-$ $2(\lambda+1) \alpha+\lambda$ and rational functions $f, g$ and $h$ by

$$
f=\frac{X-\alpha Z}{Z}, \quad g=\frac{4 \beta^{2}(X-\alpha Z)}{2 \beta(Y-\beta Z)-\mu(X-\alpha Z)}, \quad h=\frac{4 \beta^{2}(X-\alpha Z)}{-2 \beta(Y+\beta Z)-\mu(X-\alpha Z)} .
$$

Then $f$ is an element of $H^{0}\left(\tilde{E}, \mathcal{O}_{\tilde{E}}(3 o-[o])\right)$ and we have $g=T_{o^{\prime \prime}}^{*} f$ and $h=T_{o^{\prime}}^{*} f$. The following lemma is essentially due to Takahashi [9].

Lemma 1.4 (Takahashi $\left[9\right.$, p. 286]). We define five sections of $H^{0}\left(\boldsymbol{P}_{\tilde{E}}, \mathcal{O}_{\boldsymbol{P}_{\tilde{E}}}(4 \tilde{H}-\right.$ $\left.\left.\tilde{p}^{*}[o]\right)\right)^{G}$ as

$$
\begin{aligned}
& \Psi_{1}=f Z_{0}^{4}+g Z_{1}^{4}+h Z_{2}^{4}, \\
& \Psi_{2}=Z_{0} Z_{1} Z_{2}\left(Z_{0}+Z_{1}+Z_{2}\right), \\
& \Psi_{3}=g Z_{0} Z_{1}^{3}+h Z_{1} Z_{2}^{3}+f Z_{0}^{3} Z_{2}, \\
& \Psi_{4}=h Z_{0} Z_{2}^{3}+f Z_{0}^{3} Z_{1}+g Z_{1}^{3} Z_{2}, \\
& \Psi_{5}=g h Z_{1}^{2} Z_{2}^{2}+f h Z_{0}^{2} Z_{2}^{2}+f g Z_{0}^{2} Z_{1}^{2} .
\end{aligned}
$$

Then $\left\{\Psi_{1}, \Psi_{2}, \Psi_{3}, \Psi_{4}, \Psi_{5}\right\}$ is a basis of $H^{0}\left(\boldsymbol{P}_{\tilde{E}}, \mathcal{O}_{\boldsymbol{P}_{\tilde{E}}}\left(4 \tilde{H}-\tilde{p}^{*}[o]\right)\right)^{G}$.

Proof. Let $\Psi$ be an element of $H^{0}\left(\boldsymbol{P}_{\tilde{E}}, \mathcal{O}_{\boldsymbol{P}_{\tilde{E}}}\left(4 \tilde{H}-\tilde{p}^{*}[o]\right)\right)^{G}$. Since $\Psi \in H^{0}\left(\boldsymbol{P}_{\tilde{E}}\right.$, $\left.\mathcal{O}_{\boldsymbol{P}_{\tilde{E}}}\left(4 \tilde{H}-\tilde{p}^{*}[o]\right)\right), \Psi$ can be written as

$$
\Psi=\sum_{\substack{i, j, k \geq 0 \\ i+j+k=4}} \psi_{i j k} Z_{0}^{i} Z_{1}^{j} Z_{2}^{k}, \quad \psi_{i j k} \in H^{0}\left(\tilde{E}, \mathcal{O}_{\tilde{E}}\left(i o+j o^{\prime}+k o^{\prime \prime}-[o]\right)\right) .
$$

Since we have

$$
T_{o^{\prime \prime}}^{*} \Psi=\sum_{\substack{i, j, k \geq 0 \\ i+j+k=4}} T_{o^{\prime \prime}}^{*} \psi_{i j k} Z_{1}^{i} Z_{2}^{j} Z_{0}^{k},
$$

we see that $\Psi \in H^{0}\left(\boldsymbol{P}_{\tilde{E}}, \mathcal{O}_{P_{\tilde{E}}}\left(4 \tilde{H}-\tilde{p}^{*}[o]\right)\right)^{G}$ if and only if $\psi_{i j k}=T_{o^{\prime \prime}}^{*} \psi_{j k i}$ for all integers $i, j, k \geq 0$ with $i+j+k=4$. We have $\psi_{400} \in H^{0}\left(\tilde{E}, \mathcal{O}_{\tilde{E}}(4 o-[o])\right)$ and $\operatorname{dim} H^{0}\left(\tilde{E}, \mathcal{O}_{\tilde{E}}(4 o-\right.$ $[o]))=1$. Since $f$ is a non-zero element of $H^{0}\left(\tilde{E}, \mathcal{O}_{\tilde{E}}(4 o-[o])\right)$, we can write $\psi_{400}=$ $a_{1} f\left(a_{1} \in \boldsymbol{C}\right)$. Since we have $g=T_{o^{\prime \prime}}^{*} f, h=T_{o^{\prime \prime}}^{*} g$, we obtain conditions $\psi_{040}=a_{1} g$ and 
$\psi_{004}=a_{1} h$. Similarly, we obtain the conditions

$$
\begin{gathered}
\psi_{211}=\psi_{121}=\psi_{112}=a_{2}, \\
\psi_{301}=a_{3} f, \quad \psi_{130}=a_{3} g, \quad \psi_{013}=a_{3} h, \\
\psi_{310}=a_{4} f, \quad \psi_{031}=a_{4} g, \quad \psi_{103}=a_{4} h, \\
\psi_{220}=a_{5} f g, \quad \psi_{202}=a_{5} f h, \quad \psi_{022}=a_{5} g h,
\end{gathered}
$$

where $a_{i} \in \boldsymbol{C}$ for $i=2,3,4,5$. Therefore, we can write

$$
\begin{aligned}
\Psi= & a_{1}\left(f Z_{0}^{4}+g Z_{1}^{4}+h Z_{2}^{4}\right)+a_{2} Z_{0} Z_{1} Z_{2}\left(Z_{0}+Z_{1}+Z_{2}\right)+a_{3}\left(g Z_{0} Z_{1}^{3}+h Z_{1} Z_{2}^{3}+f Z_{0}^{3} Z_{2}\right) \\
& +a_{4}\left(h Z_{0} Z_{2}^{3}+f Z_{0}^{3} Z_{1}+g Z_{1}^{3} Z_{2}\right)+a_{5}\left(g h Z_{1}^{2} Z_{2}^{2}+f h Z_{0}^{2} Z_{2}^{2}+f g Z_{0}^{2} Z_{1}^{2}\right) .
\end{aligned}
$$

REMARK 1.5. By an easy calculation, we have $f g h=-4 \beta^{2}$.

REMARK 1.6. Let $\tilde{S}$ be a member in $\mathcal{U}$ which has at worst rational double points. The minimal model $S^{*}$ of $\tilde{S}$ satisfies $p_{g}\left(S^{*}\right)=3, q\left(S^{*}\right)=1, K_{S^{*}}^{2}=9$ and $\chi_{\text {top }}\left(S^{*}\right)=27$ by [9, Proposition 2.3]. Let $\tilde{S}_{P}$ be the fiber of $\tilde{p}$ at $P \in \tilde{E}$. Applying the equality (1) to $\left.\tilde{p}\right|_{\tilde{S}}: \tilde{S} \rightarrow \tilde{E}$, we obtain the equality

$$
\sum_{P \in E}\left(\chi_{\text {top }}\left(\tilde{S}_{P}\right)+4\right)+\sum_{Q \in \tilde{S}} r(Q)=27 .
$$

For any member $\tilde{S}$ in $\mathcal{U}, G$ acts on $\tilde{S}$ without fixed point. So we obtain the unramified morphism $\left.\Phi\right|_{\tilde{S}}: \tilde{S} \rightarrow \tilde{S} / G$ of degree three. Let $(\tilde{S} / G)_{Q}$ be the fiber of $\tilde{S} / G$ at $Q \in E$. If $P \in \tilde{E}$ satisfies $\varphi(P)=Q$, then the three fibers $\tilde{S}_{P}, \tilde{S}_{P^{\prime}}, \tilde{S}_{P^{\prime \prime}}$ are isomorphic to $(\tilde{S} / G)_{Q}$. Furthermore, the three analytic local rings $\mathcal{O}_{\tilde{S}, P}^{\text {an }}, \mathcal{O}_{\tilde{S}, P^{\prime}}^{\text {an }}, \mathcal{O}_{\tilde{S}, P^{\prime \prime}}^{\text {an }}$ are isomorphic to $\mathcal{O}_{\tilde{S} / G, Q}^{\text {an }}$. Thus, in order to find the Catanese-Ciliberto surface $S$ of type I, it suffices to find a nonsingular member $\tilde{S}$ in $\mathcal{U}$ which only has three singular fibers.

Remark 1.7. We assume that $\tilde{S} \in \mathcal{U}$ is defined by $\Psi \in H^{0}\left(\boldsymbol{P}_{\tilde{E}}, \mathcal{O}_{\boldsymbol{P}_{\tilde{E}}}\left(4 \tilde{H}-\tilde{p}^{*}[o]\right)\right)$. Let $U_{o} \subset \tilde{E}$ be a neighborhood of $o$. Set $t=X / Y$, which is a parameter of $\tilde{E}$ at $U_{o}$. Set $s=Z / Y$ and $u=t^{3} / s=1+(\lambda+1) t^{2}-\lambda t^{4} u^{-1}$. We may write

$$
\begin{aligned}
f=\frac{X-\alpha Z}{Z} & =\frac{t-\alpha s}{s}=\frac{t-\alpha u^{-1} t^{3}}{u^{-1} t^{3}}=\frac{u-\alpha t^{2}}{t^{2}} \\
& =\frac{1+(\lambda+1-\alpha) t^{2}+(\text { higher terms })}{t^{2}} \\
g & =\frac{4 \beta^{2}\left(u t-\alpha t^{3}\right)}{2 \beta\left(u-\beta t^{3}\right)-\mu\left(u t-\alpha t^{3}\right)}=2 \beta t+\mu t^{2}+(\text { higher terms) }, \\
h & =\frac{4 \beta^{2}\left(u t-\alpha t^{3}\right)}{-2 \beta\left(u+\beta t^{3}\right)-\mu\left(u t-\alpha t^{3}\right)}=-2 \beta t+\mu t^{2}+\text { (higher terms) } .
\end{aligned}
$$

Since $Z_{0} \in H^{0}\left(\boldsymbol{P}_{\tilde{E}}, \mathcal{O}_{\tilde{E}}\left(\tilde{H}-\tilde{F}_{o}\right)\right),\left(Z_{0}^{\prime}: Z_{1}: Z_{2}\right)=\left(t^{-1} Z_{0}: Z_{1}: Z_{2}\right)$ is a relative homogeneous coordinate system of $\tilde{p}^{-1}\left(U_{o}\right)$. Thus, the neighborhood $\tilde{p}^{-1}\left(U_{o}\right)$ of the fiber of $\tilde{S}$ at $o$ is defined by $t^{-1} \Psi\left(t Z_{0}^{\prime}, Z_{1}, Z_{2}\right)=0$. The sections $\Psi_{1}, \Psi_{2}, \Psi_{3}, \Psi_{4}, \Psi_{5}$ are written 
at $o$ as follows:

$$
\begin{aligned}
t^{-1} \Psi_{1}\left(t Z_{0}^{\prime}, Z_{1}, Z_{2}\right)= & 2 \beta\left(Z_{1}^{4}-Z_{2}^{4}\right)+t\left(Z_{0}^{\prime 4}+\mu Z_{1}^{4}+\mu Z_{2}^{4}\right)+(\text { higher terms }), \\
t^{-1} \Psi_{2}\left(t Z_{0}^{\prime}, Z_{1}, Z_{2}\right)= & Z_{0}^{\prime} Z_{1} Z_{2}\left(Z_{1}+Z_{2}\right)+t Z_{0}^{\prime 2} Z_{1} Z_{2} \\
t^{-1} \Psi_{3}\left(t Z_{0}^{\prime}, Z_{1}, Z_{2}\right)= & Z_{0}^{\prime 3} Z_{2}-2 \beta Z_{1} Z_{2}^{3}+\left(\mu Z_{1} Z_{2}^{3}+2 \beta Z_{0}^{\prime} Z_{1}^{3}\right) t+\text { (higher terms) } \\
t^{-1} \Psi_{4}\left(t Z_{0}^{\prime}, Z_{1}, Z_{2}\right)= & Z_{0}^{\prime 3} Z_{1}+2 \beta Z_{1}^{3} Z_{2}+\left(\mu Z_{1}^{3} Z_{2}-2 \beta Z_{0}^{\prime} Z_{2}^{3}\right) t+(\text { higher terms }), \\
t^{-1} \Psi_{5}\left(t Z_{0}^{\prime}, Z_{1}, Z_{2}\right)= & 2 \beta Z_{0}^{\prime 2}\left(Z_{1}^{2}-Z_{2}^{2}\right)+\left(\mu Z_{0}^{\prime 2} Z_{2}^{2}+\mu Z_{0}^{\prime 2} Z_{1}^{2}-4 \beta^{2} Z_{1}^{2} Z_{2}^{2}\right) t \\
& +(\text { higher terms }) .
\end{aligned}
$$

In particular, the defining polynomial of the fiber at $o$ is given by the constant term with respect to $t$. Furthermore, the defining polynomials at $o^{\prime}$ and $o^{\prime \prime}$ are essentially equal to that of $o$. Actually, they are permutations of indices.

2. Surface with unique singular fiber. As we saw in the previous section, the problem to find a non-singular surface $S^{\prime} \in\left|4 H-F_{0_{\tilde{E}}}\right|$ with a unique singular fiber is reduced to finding a non-singular surface $\tilde{S}$ in $\mathcal{U}$ with only three singular fibers.

2.1. The Euler contribution of a quartic curve. In our construction of surfaces, we have to consider the families of quartic curves in a projective plane. We know from the equality (4) that, if there exists a non-singular surface in $\mathcal{U}$ with only three singular fibers, then the Euler contribution of each singular fiber is nine. So by calculating the Euler number of singular quartic curves, we determine singular fibers of a non-singular surface in $\mathcal{U}$ which only has three singular fibers. Let $F$ be an irreducible reduced quartic curve in $\boldsymbol{P}^{2}$. We assume that $F$ has a singular point $P$. In order to calculate the Euler number of singular quartic curves, we first describe the classification of singular points of $F$. Let $m_{P}$ be the multiplicity of $F$ at $P$ and $s_{P}$ the number of irreducible branches at $P$. Let $v: \tilde{F} \rightarrow F$ be the normalization morphism of $F$. Set $\delta_{P}=$ length $v_{*} \mathcal{O}_{\tilde{F}, P} / \mathcal{O}_{F, P}$. In Table 1, we list the types of singularities of irreducible reduced quartic curves in terms of $m_{P}, s_{P}$ and $\delta_{P}$ (cf. [7, p. 123]). Let $\chi_{\text {top }}(F)$ be the Euler number of $F$. The possibilities of the triple $\left(m_{P}, s_{P}, \delta_{P}\right)$ are classified into nine types in Table 1. The possibilities of singularities and the Euler number of an irreducible quartic curve are classified in Table 2.

Let $Q$ be a non-reduced or reducible quartic curve. If we know the multiplicities of irreducible components of $Q$ and their configurations, we can calculate the Euler number of $Q$. We see this in Table 3. In Table 3, $L_{i}$ represent distinct lines and $D_{i}$ represent distinct conics. $A_{1}$ represents a non-singular cubic curve. $A_{2}$ and $A_{3}$ represent a cubic curve with a node $o_{2}$ and that with a cusp $c_{2}$, respectively. In Table 3, coefficients of $L_{i}$ and $D_{i}$ are multiplicities of them. We set $L_{i} \cap L_{j}=\left\{x_{i j}\right\}, L_{i} \cap D_{j}=\left\{y_{i j}^{(k)}\right\}_{k=1,2}, L_{i} \cap A_{j}=\left\{z_{i j}^{(k)}\right\}_{k=1,2,3}$ and $D_{i} \cap D_{j}=\left\{w_{i j}^{(k)}\right\}_{k=1,2,3,4}$.

In Table 3, the condition $y_{i 1}^{(1)}=y_{i 1}^{(2)}$ means that $L_{i}$ is the tangent line of $D_{1}$ at $y_{11}^{(1)}$. The condition $z_{1 j}^{(1)}=z_{1 j}^{(2)}$ means that $L_{1}$ is the tangent line of $A_{j}$ at a non-singular point $z_{1 j}^{(1)}$. 
TABLE 1. Singularities of quartics.

\begin{tabular}{cccc}
\hline Type & $m_{P}$ & $s_{P}$ & $\delta_{P}$ \\
\hline$O_{2}$ & 2 & 2 & 1 \\
$C_{2}$ & 2 & 1 & 1 \\
$O_{2}^{\prime}$ & 2 & 2 & 2 \\
$O_{2}^{\prime \prime}$ & 2 & 2 & 3 \\
$O_{3}$ & 3 & 3 & 3 \\
$C_{2}^{\prime}$ & 2 & 1 & 2 \\
$C_{2}^{\prime \prime}$ & 2 & 1 & 3 \\
$C_{3}$ & 3 & 1 & 3 \\
$C O$ & 3 & 2 & 3 \\
\hline
\end{tabular}

TABLE 2. Irreducible and reduced quartics.

\begin{tabular}{|c|c|c|c|}
\hline Number of singularities & Type of singularities & Genus of $\tilde{F}$ & $\chi_{\text {top }}(F)$ \\
\hline \multirow[t]{5}{*}{1} & $C_{2}^{\prime \prime}$ & 0 & 2 \\
\hline & $C_{3}$ & & \\
\hline & $O_{2}^{\prime \prime}$ & & 1 \\
\hline & $\mathrm{CO}$ & & \\
\hline & $\mathrm{O}_{3}$ & & 0 \\
\hline \multirow[t]{4}{*}{2} & $C_{2}^{\prime}, C_{2}$ & & 2 \\
\hline & $\mathrm{O}_{2}, \mathrm{C}_{2}^{\prime}$ & & 1 \\
\hline & $O_{2}^{\prime}, C_{2}$ & & \\
\hline & $\mathrm{O}_{2}, \mathrm{O}_{2}^{\prime}$ & & 0 \\
\hline \multirow[t]{4}{*}{3} & $C_{2}, C_{2}, C_{2}$ & & 2 \\
\hline & $C_{2}, C_{2}, O_{2}$ & & 1 \\
\hline & $C_{2}, O_{2}, O_{2}$ & & 0 \\
\hline & $\mathrm{O}_{2}, \mathrm{O}_{2}, \mathrm{O}_{2}$ & & -1 \\
\hline \multirow[t]{2}{*}{1} & $C_{2}^{\prime}$ & 1 & 0 \\
\hline & $O_{2}^{\prime}$ & & -1 \\
\hline \multirow[t]{3}{*}{2} & $C_{2}, C_{2}$ & & 0 \\
\hline & $\mathrm{C}_{2}, \mathrm{O}_{2}$ & & -1 \\
\hline & $\mathrm{O}_{2}, \mathrm{O}_{2}$ & & -2 \\
\hline \multirow[t]{2}{*}{1} & $C_{2}$ & 2 & -2 \\
\hline & $\mathrm{O}_{2}$ & & -3 \\
\hline
\end{tabular}


TABLE 3. Non-reduced or reducible quaritics.

\begin{tabular}{|c|c|c|c|}
\hline & Components of $Q$ & Intersection points & $\chi_{\text {top }}(Q)$ \\
\hline$(1)$ & $L_{1}, L_{2}, L_{3}, L_{4}$ & $x_{i j}$ are distinct points. & 2 \\
\hline (2) & $4 L_{1}$ & & 2 \\
\hline (3) & $L_{1}, L_{2}, L_{3}, L_{4}$ & $x_{12}=x_{13}=x_{23} \cdot x_{12}, x_{14}, x_{24}$ and $x_{34}$ are distinct points. & 3 \\
\hline (4) & $L_{1}, L_{2}, 2 L_{3}$ & $x_{i j}$ are distinct points. & 3 \\
\hline$(5)$ & $L_{1}, 3 L_{2}$ & $x_{i j}$ are distinct points. & 3 \\
\hline (6) & $2 L_{1}, 2 L_{2}$ & & 3 \\
\hline (7) & $L_{1}, L_{2}, 2 L_{3}$ & $x_{12}=x_{13}=x_{23}$ & 4 \\
\hline$(8)$ & $L_{1}, L_{2}, L_{3}, L_{4}$ & $x_{12}=x_{13}=x_{14}=x_{23}=x_{24}=x_{34}$ & 5 \\
\hline (9) & $L_{1}, L_{2}, D_{1}$ & $y_{i 1}^{(k)}$ are distinct points. & 1 \\
\hline (10) & $2 L_{1}, D_{1}$ & $y_{11}^{(k)}$ are distinct points. & 2 \\
\hline (11) & $L_{1}, L_{2}, D_{1}$ & $y_{11}^{(1)}=y_{11}^{(2)} \cdot y_{11}^{(1)}$ and $y_{21}^{(k)}$ are distinct points. & 2 \\
\hline (12) & $L_{1}, L_{2}, D_{1}$ & $y_{11}^{(1)}=y_{21}^{(1)} \cdot y_{11}^{(1)}$ and $y_{i 1}^{(2)}$ are distinct points. & 2 \\
\hline (13) & $L_{1}, L_{2}, D_{1}$ & $y_{11}^{(1)}=y_{11}^{(2)}, y_{21}^{(1)}=y_{21}^{(2)} \cdot y_{11}^{(1)}$ and $y_{21}^{(1)}$ are distinct points. & 3 \\
\hline (14) & $L_{1}, L_{2}, D_{1}$ & $y_{11}^{(1)}=y_{11}^{(2)}=y_{21}^{(1)} \cdot y_{11}^{(1)}$ and $y_{12}^{2}$ are distinct points. & 3 \\
\hline (15) & $2 L_{1}, D_{1}$ & $y_{11}^{(1)}=y_{11}^{(2)}$ & 3 \\
\hline (16) & $L_{1}, A_{1}$ & $z_{11}^{(k)}$ are distinct points. & -1 \\
\hline (17) & $L_{1}, A_{1}$ & $z_{11}^{(1)}=z_{11}^{(2)} \cdot z_{11}^{(1)}$ and $z_{11}^{(3)}$ are distinct points. & 0 \\
\hline (18) & $L_{1}, A_{1}$ & $z_{11}^{(1)}=z_{11}^{(2)}=z_{11}^{(3)}$ & 1 \\
\hline (19) & $L_{1}, A_{2}$ & $z_{12}^{(k)}$ are distinct points. & 0 \\
\hline (20) & $L_{1}, A_{2}$ & $z_{12}^{(1)}=z_{12}^{(2)} \cdot z_{12}^{(1)}, z_{12}^{(3)}$ and $o_{2}$ are distinct points. & 1 \\
\hline (21) & $L_{1}, A_{2}$ & $z_{12}^{(1)}=z_{12}^{(2)}=o_{2} \cdot z_{12}^{(3)}$ and $o_{2}$ are distinct points. & 1 \\
\hline (22) & $L_{1}, A_{2}$ & $z_{12}^{(1)}=z_{12}^{(2)}=z_{12}^{(3)} \cdot z_{12}^{(1)}$ and $o_{2}$ are distinct points. & 2 \\
\hline (23) & $L_{1}, A_{2}$ & $z_{12}^{(1)}=z_{12}^{(2)}=z_{12}^{(3)}=o_{2}$ & 2 \\
\hline (24) & $L_{1}, A_{3}$ & $z_{13}^{(k)}$ are distinct points. & 1 \\
\hline (25) & $L_{1}, A_{3}$ & $z_{13}^{(1)}=z_{13}^{(2)} \cdot z_{13}^{(1)}, z_{13}^{(3)}$ and $c_{2}$ are distinct points. & 2 \\
\hline (26) & $L_{1}, A_{3}$ & $z_{13}^{(1)}=z_{13}^{(2)}=c_{2} \cdot z_{13}^{(3)}$ and $c_{2}$ are distinct points. & 2 \\
\hline (27) & $L_{1}, A_{3}$ & $z_{13}^{(1)}=z_{13}^{(2)}=z_{13}^{(3)} \cdot z_{13}^{(1)}$ and $c_{2}$ are distinct points. & 3 \\
\hline (28) & $L_{1}, A_{3}$ & $z_{13}^{(1)}=z_{13}^{(2)}=z_{13}^{(3)}=c_{2}$ & 3 \\
\hline (29) & $D_{1}, D_{2}$ & $w_{12}^{(k)}$ are distinct points. & 2 \\
\hline (30) & $D_{1}, D_{2}$ & $w_{12}^{(1)}=w_{12}^{(2)} \cdot w_{12}^{(1)}, w_{12}^{(3)}$ and $w_{12}^{(4)}$ are distinct points. & 1 \\
\hline (31) & $D_{1}, D_{2}$ & $w_{12}^{(1)}=w_{12}^{(2)}, w_{12}^{(3)}=w_{12}^{(4)} \cdot w_{12}^{(1)}$ and $w_{12}^{(3)}$ are distinct points. & 2 \\
\hline (32) & $2 D_{1}$ & & 2 \\
\hline
\end{tabular}


The condition $z_{1 j}^{(1)}=z_{1 j}^{(2)}=z_{1 j}^{(3)}$ means that $L_{1}$ is the tangent line of $A_{j}$ at a flex point $z_{1 j}^{(1)}$ of $A_{j}$. The condition $z_{12}^{(1)}=z_{12}^{(2)}=o_{2}$ means that $L_{1}$ intersects $A_{2}$ at $o_{2}$. The condition $z_{12}^{(1)}=z_{12}^{(2)}=z_{12}^{(3)}=o_{2}$ means that $L_{1}$ is tangent to $A_{2}$ at $o_{2}$. The condition $z_{13}^{(1)}=z_{13}^{(2)}=c_{2}$ means that $L_{1}$ intersects $A_{3}$ at $c_{2}$. The condition $z_{13}^{(1)}=z_{13}^{(2)}=z_{13}^{(3)}=c_{2}$ means that $L_{1}$ is tangent to $A_{3}$ at $c_{2}$. The condition $w_{12}^{(i)}=w_{12}^{(i+1)}$ means that $D_{1}$ and $D_{2}$ have the common tangent line at a point $w_{12}^{(i)}$. To help to understand Table 3, we describe in Figure 1 the figures corresponding to the curves.

From Tables 2 and 3 , we see $0 \leq \chi_{\text {top }}(F)+4 \leq 9$ for any quartic curve $F$. The equality (4) implies that, if there exists a non-singular member $\tilde{S}$ in $\mathcal{U}$ with only three singular fibers $\tilde{S}_{P}, \tilde{S}_{P^{\prime}}, \tilde{S}_{P^{\prime \prime}}$, then these are four lines intersecting at one point. Tables 2 and 3 imply the following important lemma.

LEMMA 2.1. Let $S$ be a Catanese-Ciliberto surface with $g=3$. Then singular fibers of the Albanese map $a: S \rightarrow \operatorname{Alb}(S)=:$ E are reduced.

PROOF. It suffices to show that a singular fiber of $S^{\prime} \in\left|4 H-F_{0_{E}}\right|$ with at worst rational double points is reduced. We assume that $S_{P}^{\prime}$ is a singular fiber. Let $U_{P} \subset E$ be a neighborhood and $t$ a local parameter of $E$ at $U_{P}$. Let $\left(X_{0}: X_{1}: X_{2}\right)$ be a relative homogeneous coordinate of $p^{-1}\left(U_{P}\right)$. The defining polynomial of $S^{\prime}$ at $P$ is written by $\Psi=$ $\sum_{i=0}^{\infty} t^{i} \psi_{i}\left(X_{0}, X_{1}, X_{2}\right)$, where $\psi_{i}$ are homogeneous polynomial in $X_{0}, X_{1}, X_{2}$ of degree four. Since $\left.\partial_{t} \Psi\right|_{t=0}=\psi_{1}\left(X_{0}, X_{1}, X_{2}\right)$, singular points of $S^{\prime}$ satisfy $\psi_{1}\left(X_{0}, X_{1}, X_{2}\right)=0$. Let $F_{1} \subset \boldsymbol{P}^{2}$ be the curve defined by $\psi_{1}$. Suppose $S_{P}^{\prime}$ is not reduced. Then any point in a non-reduced irreducible component of $S_{P}^{\prime}$ is a singular point of $S_{P}^{\prime}$. By using Bézout's theorem, a non-reduced irreducible component of $S_{P}^{\prime}$ intersects $F_{1}$. In particular, $S^{\prime}$ is singular at this point of $S_{P}^{\prime}$. We consider two cases as follows.

(i) The case that $S_{P}^{\prime}$ contains a line $L$ with the multiplicity greater than or equal to two. If $L$ intersects $F_{1}$ at $Q$, then we can write the local equation of $S^{\prime}$ at $Q$ as follows:

$$
\begin{gathered}
\Psi=l^{2} \sum_{0 \leq i+j \leq 2} a_{i j} l^{i} y^{j}+t \sum_{0 \leq i+j \leq 4} b_{i j} l^{i} y^{j}+t^{2} \sum_{0 \leq i+j \leq 4} c_{i j} l^{i} y^{j}+\text { (higher terms) } \\
\left(a_{i j}, b_{i j}, c_{i j} \in \boldsymbol{C},\left(a_{00}, a_{01}, a_{02}\right) \neq(0,0,0), b_{00}=0\right),
\end{gathered}
$$

where $(l, y)$ is an inhomogeneous coordinate system of $A^{2}$ such that $L$ is defined by the equation $l=0$. Since $Q$ is a rational double point of $S^{\prime}$, we have $r(Q) \geq 1$. We show that the sum of the Euler contributions of the rational double points of $S^{\prime}$ is at least four in any cases.

If $L$ intersects $F_{1}$ at four points, then the sum of the Euler contributions of the rational double points of $S^{\prime}$ is at least four. Let $m_{Q}\left(L, F_{1}\right)$ be the local intersection number of $L$ and $F_{1}$ at $Q$. First assume that $m_{Q}\left(L, F_{1}\right)=2$ or 3 . Then we can assume $b_{01}=0$. By blowing up $\Psi$ at the point satisfying $t=l=y=0\left(l=l_{1} y, t=t_{1} y\right)$, we obtain the defining 


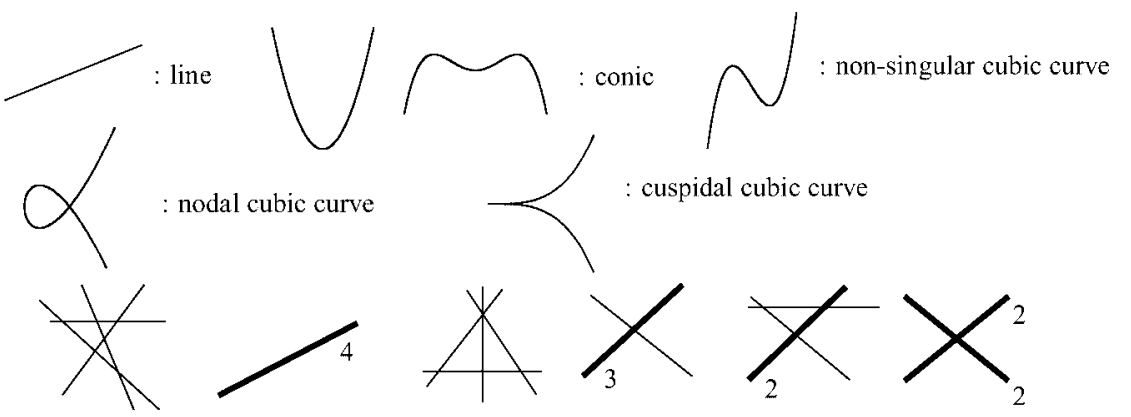

(1)

(2)

(3)

(4)

(5)

(6)

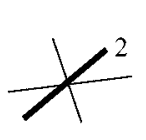

(7)

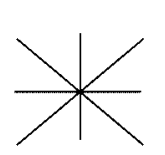

(8)

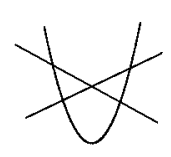

(9)

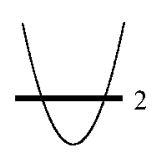

(10)

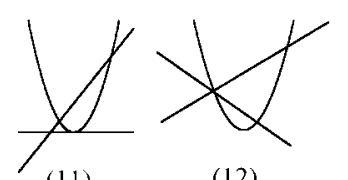

(12)

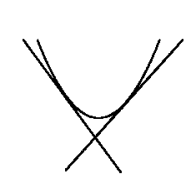

(13)

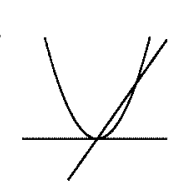

(14)

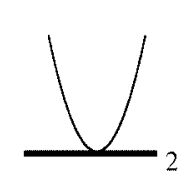

(15)

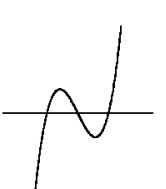

(16)

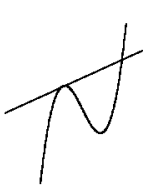

(17)

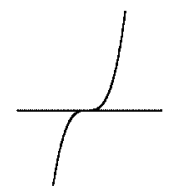

(18)

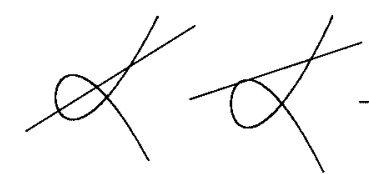

(19)

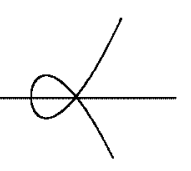

(21)

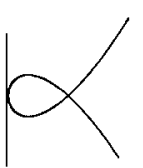

(22)

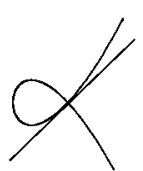

(23)

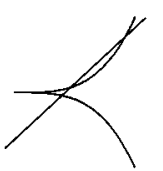

(24)

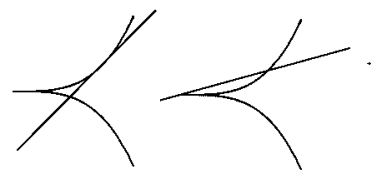

(25)

(26)

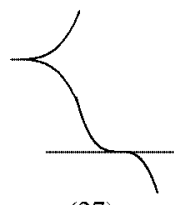

(27)

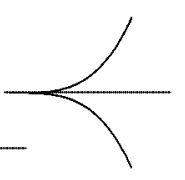

(28)

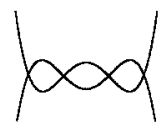

(29)<smiles>CC(C)C1CC(C)C1C</smiles>

(30)

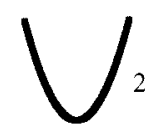

(31)<smiles>CC(C)(C)C1CC1C(C)(C)C</smiles>

(32)

FIGURE 1. Figures corresponding to curves in Table 3. Numbers with the bold curves are the multiplicities.

polynomials of the proper transform and exceptional curves as follows:

$$
\begin{gathered}
\Psi^{\prime}=l_{1}^{2} \sum_{0 \leq i+j \leq 2} a_{i j} l_{1}^{i} y^{i+j}+t_{1} \sum_{1 \leq i+j \leq 4} b_{i j} l_{1}^{i} y^{i+j-1}+t_{1}^{2} \sum_{0 \leq i+j \leq 4} c_{i j} l_{1}^{i} y^{i+j}+y \text { (higher terms), } \\
\left.\Psi^{\prime}\right|_{y=0}=a_{00} l_{1}^{2}+b_{10} l_{1} t_{1}+c_{00} t_{1}^{2} .
\end{gathered}
$$


Since we have

$$
\begin{gathered}
\left.\partial_{t_{1}} \Psi^{\prime}\right|_{y=0}=b_{10} l_{1}+2 c_{00} t_{1}, \\
\left.\partial_{l_{1}} \Psi^{\prime}\right|_{y=0}=2 a_{00} l_{1}+b_{10} t_{1}, \\
\left.\partial_{y} \Psi^{\prime}\right|_{y=0}=\left(a_{10} l_{1}+a_{01}\right) l_{1}^{2}+\left(b_{20} l_{1}^{2}+b_{11} l_{1}+b_{02}\right) t_{1}+\left(c_{01}+c_{10} l_{1}\right) t_{1}^{2}+\text { (higher terms), }
\end{gathered}
$$

the surface defined by $\Psi^{\prime}=0$ has a singular point at $t_{1}=l_{1}=0$ and we have $r(Q) \geq$ 3. Since $S_{P}^{\prime}$ has another rational double point, the sum of the Euler contributions of the rational double points of $S^{\prime}$ is at least four in this case. In the case where $m_{P}\left(L, F_{1}\right)=4$, we can show that $r(Q) \geq 4$ in a similar manner as in the previous case. Hence, we obtain $\sum_{Q} r(Q) \geq 4$. Furthermore, from Table 3, the Euler contribution of a non-reduced quartic curve which contains a multiple line is at least six. It contradicts the fact that the sum of the Euler contributions is nine. Hence, $S_{P}^{\prime}$ does not contain a line with the multiplicity greater than one.

(ii) The case that $S_{P}^{\prime}$ consists of a conic $D$ with the multiplicity two. If $D$ intersects $F_{1}$ at $Q$, then $Q$ is a rational double point of $S^{\prime}$, i.e., $r(Q) \geq 1$. If $D$ intersects $F_{1}$ at eight points, then the sum of the Euler contributions of the rational double points of $S^{\prime}$ is at least eight. Furthermore, we know the Euler contribution of $S_{P}^{\prime}$ is two by Table 3. It contradicts the fact that the sum of the Euler contributions is nine. We can show that $S_{P}^{\prime}$ does not consist of a conic with the multiplicity two similarly as in the case (i).

2.2. Defining equations of the Catanese-Ciliberto surfaces with unique singular fiber I. Let $\tilde{S}_{i}$ be the surface defined by $\Psi_{i}=0$ in $\boldsymbol{P}_{\tilde{E}}$ for $i=1,2, \ldots, 5$. We observe these surfaces first. The first part of Theorem 0.2 is proved by the following example.

EXAMPLE 2.2. The defining polynomial of the fiber of $\tilde{S}_{1}$ at $o$ is $\Psi_{1}(o)=2 \beta\left(Z_{1}^{4}-\right.$ $\left.Z_{2}^{4}\right)$. The equation $\Psi_{1}(o)=0$ defines the curve which consists of four lines intersecting at one point. The fibers at $o^{\prime}$ and $o^{\prime \prime}$ are isomorphic to the fiber at $o$. Since we have

$$
\partial_{Z_{0}} \Psi_{1}=4 f Z_{0}^{3}, \quad \partial_{Z_{1}} \Psi_{1}=4 g Z_{1}^{3}, \quad \partial_{Z_{2}} \Psi_{1}=4 h Z_{2}^{3}
$$

and $f, g$ and $h$ are non-zero regular on $\tilde{E} \backslash\left\{o, o^{\prime}, o^{\prime \prime}\right\}$, other fibers are non-singular. If $\tilde{S}_{1}$ has singular points, then one of them is contained in the singular fiber at $o$. However, since the local defining polynomial of $\tilde{S}_{1}$ at $o$ is

$$
t^{-1} \Psi_{1}\left(t Z_{0}^{\prime}, Z_{1}, Z_{2}\right)=2 \beta\left(Z_{1}^{4}-Z_{2}^{4}\right)+t\left(Z_{0}^{\prime 4}+\mu Z_{1}^{4}+\mu Z_{2}^{4}\right)+\text { (higher terms) },
$$

$\tilde{S}_{1}$ is non-singular. Hence, we obtain the Catanese-Ciliberto surface $S_{1}=\tilde{S}_{1} / G$ of type I.

EXAmple 2.3. Since almost all fibers of $\tilde{S}_{2}$ consists of four lines, $S_{2}=\tilde{S}_{2} / G$ is not normal. In particular, it is not a Catanese-Ciliberto surface.

EXAMPLE 2.4. The defining polynomial of the fiber of $\tilde{S}_{3}$ at $o$ is $\Psi_{3}(o)=$ $Z_{2}\left(-2 \beta Z_{1} Z_{2}^{2}+Z_{0}^{3}\right)$. Since $\beta$ is not zero, the fiber of $\tilde{S}_{3}$ at $o$ is the union of a cuspidal cubic curve and the tangent line at the cusp. Since there exists no point of $\tilde{S}_{3}$ satisfying the 
equations

$$
\begin{aligned}
& \partial_{Z_{0}} \Psi_{3}=g Z_{1}^{3}+3 f Z_{0}^{2} Z_{2}=0, \quad \partial_{Z_{1}} \Psi_{3}=h Z_{2}^{3}+3 g Z_{0} Z_{1}^{2}=0, \\
& \partial_{Z_{2}} \Psi_{3}=f Z_{0}^{3}+3 h Z_{1} Z_{2}^{2}=0,
\end{aligned}
$$

$\tilde{S}_{3}$ has no other singular fibers. Because the local defining polynomial of $\tilde{S}_{3}$ at $o$ is

$$
t^{-1} \Psi_{3}\left(t Z_{0}^{\prime}, Z_{1}, Z_{2}\right)=-2 \beta Z_{1} Z_{2}^{3}+Z_{0}^{\prime 3} Z_{2}+\left(2 \beta Z_{0}^{\prime} Z_{1}^{3}+\mu Z_{1} Z_{2}^{3}\right) t+\text { (higher terms), }
$$

$\tilde{S}_{3}$ has a rational double point of type $A_{2}$ at $(o,(0: 1: 0))$. Hence, it has the same singularities over $o^{\prime}$ and $o^{\prime \prime}$.

We define the involution $\iota: \tilde{E} \rightarrow \tilde{E}$ of the elliptic curve $\tilde{E}$ by $\iota(P)=-P$. This involution is lifted to those of $\boldsymbol{P}_{\tilde{E}}$ and $\mathcal{O}_{\boldsymbol{P}_{\tilde{E}}}(\tilde{H})$ so that $Z_{0}, Z_{1}, Z_{2}$ are mapped to $Z_{0}, Z_{2}, Z_{1}$, respectively. We denote this involution by $\bar{\imath}$.

REMARK 2.5. We see that $\iota^{*} f=f, \iota^{*} g=h$ and $\iota^{*} h=g$. By these equalities, the automorphism $\bar{\imath}$ induces an isomorphism $\left.\bar{\imath}\right|_{\tilde{S}_{4}}: \tilde{S}_{4} \stackrel{\sim}{\rightarrow} \tilde{S}_{3}$ of divisors of $\boldsymbol{P}_{\tilde{E}}$.

EXAMPLE 2.6. Since all fibers of $\tilde{S}_{5}$ have singular points $(0: 0: 1),(0: 1: 0)$, $(1: 0: 0), S_{5}=\tilde{S}_{5} / G$ is not a Catanese-Ciliberto surface.

The automorphism $\bar{\imath}$ induces an involution of $\tilde{S}_{1}$. Let $\tilde{S} \in \mathcal{U}$ be a surface with at most rational double points defined by $\Psi=\sum_{i=1}^{5} a_{i} \Psi_{i} \in H^{0}\left(\boldsymbol{P}_{\tilde{E}}, \mathcal{O}_{\boldsymbol{P}_{\tilde{E}}}\left(4 \tilde{H}-\tilde{p}^{*}[o]\right)\right)^{G}$. In the following remark, we note some properties of $\tilde{S} \in \mathcal{U}$ with an involution which need later.

REMARK 2.7. If we assume $a_{3}=a_{4}$, then $\left.\bar{\imath}\right|_{\tilde{S}}$ gives an automorphism of $\tilde{S}$ with order two, because we have $\imath^{*} \Psi_{i}=\Psi_{i}(i=1,2,5)$ and $\imath^{*} \Psi_{3}=\Psi_{4}, \bar{\imath}^{*} \Psi_{4}=\Psi_{3}$.

(i) Each of fibers at $o, o^{\prime}$ and $o^{\prime \prime}$ contains a line. The fiber at $o$ contains the line defined by $Z_{1}+Z_{2}=0$. Hence, these fibers are reducible singular quartic curves.

(ii) We assume that $\tilde{S}_{P}$ has a singular point $Q=\left(q_{0}: q_{1}: q_{2}\right)$ for a point $P$ on $\tilde{E} \backslash\left\{o, o^{\prime}, o^{\prime \prime}\right\}$. Let $\Psi(P)$ be the defining polynomial of the fiber of $\tilde{S}$ at $P$. Since we have $f(P)=f(-P), g(P)=h(-P)$ and $h(P)=g(-P)$, we obtain $\Psi(P)\left(Z_{0}, Z_{1}, Z_{2}\right)=$ $\Psi(-P)\left(Z_{0}, Z_{2}, Z_{1}\right)$. Therefore, $\tilde{S}_{-P}$ has a singular point $\left(q_{0}: q_{2}: q_{1}\right)$.

(iii) Let $\gamma_{1}, \gamma_{2}, \gamma_{3} \in \tilde{E}$ be points of order two. If $\tilde{S}_{P}$ is a singular fiber, then $\tilde{S}_{P^{\prime}}, \tilde{S}_{P^{\prime \prime}}$, $\tilde{S}_{-P}, \tilde{S}_{-P^{\prime}}$ and $\tilde{S}_{-P^{\prime \prime}}$ are also singular fibers since the group $G$ of order three is acting on $\tilde{S}$. Set $\Gamma=\left\{o, o^{\prime}, o^{\prime \prime}, \gamma_{1}, \gamma_{1}^{\prime}, \gamma_{1}^{\prime \prime}, \gamma_{2}, \gamma_{2}^{\prime}, \gamma_{2}^{\prime \prime}, \gamma_{3}, \gamma_{3}^{\prime}, \gamma_{3}^{\prime \prime}\right\}$. If $P \in \tilde{E} \backslash \Gamma$, the number of elements of the set $\left\{P, P^{\prime}, P^{\prime \prime},-P,-P^{\prime},-P^{\prime \prime}\right\}$ is six, i.e., $\tilde{S}$ has six singular fibers which are isomorphic to each other.

(iv) Similarly, if $\tilde{S}$ has a singular point in the fiber at $P \in \tilde{E} \backslash \Gamma$, then $\tilde{S}$ has five other singular points. Moreover, these analytic local rings are isomorphic to each other.

As we saw in Example 2.2, $S_{1}=\tilde{S}_{1} / G$ is the Catanese-Ciliberto surface of type I. The following proposition implies that there are at most three other possibilities for each $E$. 
PROPOSITION 2.8. For an arbitrary elliptic curve E, let $S$ be a Catanese-Ciliberto surface of type I with $\mathrm{Alb}(S) \cong E$. Let $\varphi: \tilde{E} \rightarrow E$ be an isogeny of degree three and $\zeta_{1}, \zeta_{2}, \zeta_{3}$ the cubic roots of $-2 \beta$. Then the pull-back of $S$ by the unramified finite morphism $\Phi: \boldsymbol{P}_{\tilde{E}} \rightarrow$ $\boldsymbol{P}_{E}$ of degree three induced by $\varphi$ is defined by one of the four equations $\Psi_{1}, \Psi_{\zeta_{1}}, \Psi_{\zeta_{2}}, \Psi_{\zeta_{3}}$, where

$$
\begin{aligned}
\Psi_{1}= & f Z_{0}^{4}+g Z_{1}^{4}+h Z_{2}^{4}=0, \\
\Psi_{\zeta}= & f Z_{0}^{4}+g Z_{1}^{4}+h Z_{2}^{4}-12 \zeta^{2} Z_{0} Z_{1} Z_{2}\left(Z_{0}+Z_{1}+Z_{2}\right) \\
& +4\left(g Z_{0} Z_{1}^{3}+h Z_{1} Z_{2}^{3}+f Z_{0}^{3} Z_{2}\right)+4\left(h Z_{0} Z_{2}^{3}+f Z_{0}^{3} Z_{1}+g Z_{1}^{3} Z_{2}\right) \\
& -6 \zeta^{-2}\left(g h Z_{1}^{2} Z_{2}^{2}+f h Z_{0}^{2} Z_{2}^{2}+f g Z_{0}^{2} Z_{1}^{2}\right)=0 \text { for } \zeta=\zeta_{1}, \zeta_{2}, \zeta_{3} .
\end{aligned}
$$

Proof. Let $\tilde{S}$ be a non-singular surface in $\mathcal{U}$ with only three singular fibers. We prove that $\tilde{S}$ is defined by $\Psi_{1}=0$ or $\Psi_{\zeta}=0$ for a cubic root $\zeta$ of $-2 \beta$. Let $\Psi$ be defining equation of $\tilde{S}$ in $\boldsymbol{P}_{\tilde{E}}$. Then $\Psi$ can be written as

$$
\begin{aligned}
\Psi= & a_{1}\left(f Z_{0}^{4}+g Z_{1}^{4}+h Z_{2}^{4}\right)+a_{2} Z_{0} Z_{1} Z_{2}\left(Z_{0}+Z_{1}+Z_{2}\right) \\
& +a_{3}\left(g Z_{0} Z_{1}^{3}+h Z_{1} Z_{2}^{3}+f Z_{0}^{3} Z_{2}\right)+a_{4}\left(h Z_{0} Z_{2}^{3}+f Z_{0}^{3} Z_{1}+g Z_{1}^{3} Z_{2}\right) \\
& +a_{5}\left(g h Z_{1}^{2} Z_{2}^{2}+f h Z_{0}^{2} Z_{2}^{2}+f g Z_{0}^{2} Z_{1}^{2}\right)
\end{aligned}
$$

A singular fiber of $\tilde{S}$ consists of four lines intersecting at one point. We assume that $\tilde{S}$ has a singular fiber at $P \in \tilde{E}$ and $\tilde{S}_{P}$ consists of four lines intersecting at a point $Q=\left(q_{0}: q_{1}: q_{2}\right)$.

(i) The case that $P$ is other than $o, o^{\prime}$ and $o^{\prime \prime}$. Since $\Psi$ is $G$-invariant, $\tilde{S}_{P^{\prime}}$ and $\tilde{S}_{P^{\prime \prime}}$ are also quartic curves which consist of four lines intersecting at one point. We may assume $q_{2}=1$ by replacing $P$ by $P^{\prime}$ or $P^{\prime \prime}$, if necessary. Denote $f=f(P), g=g(P)$ and $h=h(P)$. Since $m_{Q}\left(\tilde{S}_{P}\right)=4, Q$ satisfies the following equations:

$$
\begin{gathered}
\partial_{Z_{0}} \partial_{Z_{0}} \partial_{Z_{0}} \Psi(P)=6 a_{3} f+24 a_{1} f q_{0}+6 a_{4} f q_{1}=0, \\
\partial_{Z_{0}} \partial_{Z_{0}} \partial_{Z_{1}} \Psi(P)=2 a_{2}+6 a_{4} f q_{0}+4 a_{5} f g q_{1}=0, \\
\partial_{Z_{0}} \partial_{Z_{0}} \partial_{Z_{2}} \Psi(P)=4 a_{5} f h+6 a_{3} f q_{0}+2 a_{2} q_{1}=0, \\
\partial_{Z_{0}} \partial_{Z_{1}} \partial_{Z_{1}} \Psi(P)=2 a_{2}+4 a_{5} f g q_{0}+6 a_{3} g q_{1}=0, \\
\partial_{Z_{0}} \partial_{Z_{1}} \partial_{Z_{2}} \Psi(P)=2 a_{2}+2 a_{2} q_{0}+2 a_{2} q_{1}=0, \\
\partial_{Z_{0}} \partial_{Z_{2}} \partial_{Z_{2}} \Psi(P)=6 a_{4} h+4 a_{5} f h q_{0}+2 a_{2} q_{1}=0, \\
\partial_{Z_{1}} \partial_{Z_{1}} \partial_{Z_{1}} \Psi(P)=6 a_{4} g+6 a_{3} g q_{0}+24 a_{1} g q_{1}=0, \\
\partial_{Z_{1}} \partial_{Z_{1}} \partial_{Z_{2}} \Psi(P)=4 a_{5} g h+2 a_{2} q_{0}+6 a_{4} g q_{1}=0, \\
\partial_{Z_{1}} \partial_{Z_{2}} \partial_{Z_{2}} \Psi(P)=6 a_{3} h+4 a_{5} g h q_{1}+2 a_{2} q_{0}=0 .
\end{gathered}
$$

From (9), we obtain $a_{2}=0$ or $1+q_{0}+q_{1}=0$. 
(i $\left.\mathrm{i}_{1}\right)$ The case $a_{2}=0$. From (6), (7), (8), (10), (12) and (13), we obtain conditions

$$
\begin{gathered}
2 a_{5} g q_{1}+3 a_{4} q_{0}=0, \\
2 a_{5} h+3 a_{3} q_{0}=0, \\
2 a_{5} f q_{0}+3 a_{3} q_{1}=0, \\
2 a_{5} f q_{0}+3 a_{4}=0, \\
2 a_{5} h+3 a_{4} q_{1}=0, \\
2 a_{5} g q_{1}+3 a_{3}=0 .
\end{gathered}
$$

If we assume $a_{3}=0, a_{4}=0$ or $a_{5}=0$, then we obtain conditions $a_{3}=a_{4}=a_{5}=0$ and $\Psi=\Psi_{1}$. Thus we suppose $a_{3}, a_{4}, a_{5} \neq 0$, so we obtain $q_{0}=-2 a_{5} h / 3 a_{3}, q_{1}=-2 a_{5} h / 3 a_{4}$. From (14), (16), (17) and (19), we have

$$
\begin{gathered}
3 a_{4}^{2}+2 a_{3} a_{5} g=0, \\
3 a_{3}^{2}+2 a_{4} a_{5} f=0, \\
3 a_{3} a_{4}+4 a_{5}^{2} f h=0, \\
3 a_{3} a_{4}+4 a_{5}^{2} g h=0 .
\end{gathered}
$$

By taking the difference (22) - (23), we obtain the condition $f=g$. Therefore, we obtain the condition $a_{4}^{3}-a_{3}^{3}=0$ from $(20) \times a_{4}-(21) \times a_{3}$. Then $a_{4}$ is equal to $a_{3}$ or $\omega a_{3}$, where $\omega \in \boldsymbol{C}$ satisfies $\omega^{3}=1, \omega \neq 1$. If $a_{3}=a_{4}$, then $\tilde{S}$ has a singular fiber at $o$ according to Remark 2.7(i). This contradicts the fact that $\tilde{S}$ has only three singular fibers. If $a_{4}=\omega a_{3}$, then we have $q_{1}=\omega^{2} q_{0}$. From (5), (11), (21) and (22), we have the following equations:

$$
\begin{gathered}
6 a_{3} f+24 a_{1} f q_{0}+6 a_{3} f q_{0}=0, \\
6 a_{3} \omega f+6 a_{3} f q_{0}+24 a_{1} \omega^{2} f q_{0}=0, \\
3 a_{3}^{2}+2 \omega a_{3} a_{5} f=0, \\
3 a_{3}^{2} \omega+4 a_{5}^{2} f h=0 .
\end{gathered}
$$

By considering (24) $\times \omega^{2}-(25)$, we have $q_{0}=\omega^{2}$ and $q_{1}=\omega$. On the other hand, we obtain conditions $\omega^{2} a_{3}-2 a_{5} h=0$ and $q_{0}=-\omega^{2} / 3$ from $(26) \times \omega-(27)$. So (5) $-(13)$ have no common solutions for $\left(q_{0}, q_{1}\right)$, i.e., the case $a_{2}=0$ does not occur.

(i2) The case $1+q_{0}+q_{1}=0$. From (5) and (11), we have

$$
\begin{aligned}
& a_{3}+4 a_{1} q_{0}-a_{4} q_{0}-a_{4}=0, \\
& a_{4}+a_{3} q_{0}-4 a_{1} q_{0}-4 a_{1}=0 .
\end{aligned}
$$

If $a_{3}=4 a_{1}$ or $a_{4}=4 a_{1}$ holds, then we have $a_{3}=a_{4}$ by (28) and (29). Then $\tilde{S}_{o}$ is a singular fiber by Remark 2.7(i). It contradicts the fact that $\tilde{S}$ has only three singular fibers. Therefore, we have $q_{0}=\left(a_{4}-a_{3}\right) /\left(4 a_{1}-a_{4}\right)=\left(4 a_{1}-a_{4}\right) /\left(a_{3}-4 a_{1}\right)$ and $q_{1}=-q_{0}-1=$ $\left(a_{3}-4 a_{1}\right) /\left(4 a_{1}-a_{4}\right)$. Hence, we have $q_{0} q_{1}=1$. By $q_{0} q_{1}=1$ and $1+q_{0}+q_{1}=0$, we 
obtain $\left(q_{0}, q_{1}\right)=\left(\omega, \omega^{2}\right)$. We get the following equations by (6), (8), (7), (12), (10) and (13):

$$
\begin{aligned}
a_{2}+3 a_{4} f \omega+2 a_{5} f g \omega^{2}=0, & a_{2}+2 a_{5} f g \omega+3 a_{3} g \omega^{2}=0, \\
2 a_{5} f h+3 a_{3} f \omega+a_{2} \omega^{2}=0, & 2 a_{5} g h+a_{2} \omega+3 a_{4} g \omega^{2}=0, \\
3 a_{4} h+2 a_{5} f h \omega+a_{2} \omega^{2}=0, & 3 a_{3} h+2 a_{5} g h \omega^{2}+a_{2} \omega=0 .
\end{aligned}
$$

We set the matrix

$$
M=\left(\begin{array}{cccc}
1 & 0 & 3 f \omega & 2 f g \omega^{2} \\
1 & 3 g \omega^{2} & 0 & 2 f g \omega \\
\omega^{2} & 3 f \omega & 0 & 2 f h \\
\omega & 0 & 3 g \omega^{2} & 2 g h \\
\omega^{2} & 0 & 3 h & 2 f h \omega \\
\omega & 3 h & 0 & 2 g h \omega^{2}
\end{array}\right) .
$$

Let $M_{i j k l}$ be the determinant of the matrix consisting of $i, j, k, l$-rows of $M$. If $\operatorname{rank} M=4$, i.e., if $M_{i j k l} \neq 0$ for some $(i, j, k, l)$, then we obtain $a_{2}=a_{3}=a_{4}=a_{5}=0$.

By an easy calculation, we have

$$
\begin{gathered}
M_{1234}=18(f-g) f g\left(f \omega+g \omega^{2}+h\right), \\
M_{1256}=18(g-f) h\left(g h \omega+f h \omega^{2}+f g\right), \\
M_{1356}=18(f-h) f h\left(f \omega+g \omega^{2}+h\right) .
\end{gathered}
$$

If all of them are zero, then $f=g=h$. Since $g(P)=h(P), P$ is a point of order two, i.e., $P$ is one of the points $(0: 0: 1),(1: 0: 1)$ and $(\lambda: 0: 1)$. If we assume $P=(0: 0: 1)$, then we obtain $3 \alpha^{2}-4(\lambda+1) \alpha+5 \lambda=0$ from $f(P)=g(P)$. Since this and (3) are not satisfied simultaneously, $P$ is not $(0: 0: 1)$.

Similarly, $P$ is neither $(1: 0: 1)$ nor $(\lambda: 0: 1)$. Thus, $f(P)=g(P)=h(P)$ does not occur. One of (30), (31) and (32) is not zero, hence, we have $a_{2}=a_{3}=a_{4}=a_{5}=0$, i.e., $\tilde{S}=\tilde{S}_{1}$. Then this is not the case by Example 2.2. Hence, $1+q_{0}+q_{1}=0$ is also impossible. Thus, case (i) does not occur.

(ii) The case that $P$ coincides with one of $o, o^{\prime}$ and $o^{\prime \prime}$. As we saw in the Section 1.2, we may assume that $P=o$. By Remark 1.7, the defining equation of $\tilde{S}_{o}$ can be written as

$$
\begin{aligned}
\Psi(o)= & 2 \beta a_{1}\left(Z_{1}^{4}-Z_{2}^{4}\right)+a_{2} Z_{0}^{\prime} Z_{1} Z_{2}\left(Z_{1}+Z_{2}\right)+a_{3} Z_{2}\left(-2 \beta Z_{1} Z_{2}^{2}+Z_{0}^{\prime 3}\right) \\
& +a_{4} Z_{1}\left(2 \beta Z_{1}^{2} Z_{2}+Z_{0}^{\prime 3}\right)-2 \beta a_{5} Z_{0}^{\prime 2}\left(Z_{2}^{2}-Z_{1}^{2}\right)=0 .
\end{aligned}
$$


Since $m_{Q}\left(\tilde{S}_{o}\right)=4, Q$ satisfies the following equations:

$$
\begin{gathered}
\partial_{Z_{0}} \partial_{Z_{0}} \partial_{Z_{0}} \Psi(P)=6 a_{4} q_{1}+6 a_{3} q_{2}=0, \\
\partial_{Z_{0}} \partial_{Z_{0}} \partial_{Z_{1}} \Psi(P)=6 a_{4} q_{0}+8 a_{5} q_{1} \beta=0, \\
\partial_{Z_{0}} \partial_{Z_{0}} \partial_{Z_{2}} \Psi(P)=6 a_{3} q_{0}-8 a_{5} q_{2} \beta=0, \\
\partial_{Z_{0}} \partial_{Z_{1}} \partial_{Z_{1}} \Psi(P)=2 a_{2} q_{2}+8 a_{5} q_{0} \beta=0, \\
\partial_{Z_{0}} \partial_{Z_{1}} \partial_{Z_{2}} \Psi(P)=2 a_{2} q_{1}+2 a_{2} q_{2}=0, \\
\partial_{Z_{0}} \partial_{Z_{2}} \partial_{Z_{2}} \Psi(P)=2 a_{2} q_{1}-8 a_{5} q_{0} \beta=0, \\
\partial_{Z_{1}} \partial_{Z_{1}} \partial_{Z_{1}} \Psi(P)=48 a_{1} q_{1} \beta+12 a_{4} q_{2} \beta=0, \\
\partial_{Z_{1}} \partial_{Z_{1}} \partial_{Z_{2}} \Psi(P)=2 a_{2} q_{0}+12 a_{4} q_{1} \beta=0, \\
\partial_{Z_{1}} \partial_{Z_{2}} \partial_{Z_{2}} \Psi(P)=2 a_{2} q_{0}-12 a_{3} q_{2} \beta=0, \\
\partial_{Z_{2}} \partial_{Z_{2}} \partial_{Z_{2}} \Psi(P)=-12 a_{3} q_{1} \beta-48 a_{1} q_{2} \beta=0 .
\end{gathered}
$$

From (37), we have $a_{2}=0$ or $q_{1}+q_{2}=0$.

(ii ${ }_{1}$ ) The case $a_{2}=0$. We easily obtain $a_{3}=a_{4}=a_{5}=0$ by (33)-(42), i.e., $\Psi=a_{1} \Psi_{1}$. Then $\tilde{S}=\tilde{S}_{1}$ covers the Catanese-Ciliberto surface $S_{1}$ in Example 2.2.

(ii $i_{2}$ The case $q_{1}+q_{2}=0$. If we assume $q_{1}=q_{2}=0$, then we obtain $a_{2}=a_{3}=$ $a_{4}=a_{5}=0$. Hence, we can assume $-q_{1}=q_{2}=1$. By (33)-(42), we obtain conditions

$$
\begin{gathered}
a_{3}=a_{4}=4 a_{1}, \quad 3 a_{1} q_{0}-a_{5} \beta=0, \\
2 a_{2}+8 a_{5} q_{0} \beta=0, \quad a_{2} q_{0}-24 a_{1} \beta=0 .
\end{gathered}
$$

If $a_{1}=0$, then we have $a_{2}=a_{3}=a_{4}=a_{5}=0$. So we may assume $a_{1}=1$. Therefore, we obtain $a_{5}=3 q_{0} \beta^{-1}, a_{2}=-12 q_{0}^{2}$ and $q_{0}^{3}=-2 \beta$. Since $\beta \neq 0$, the equation $\zeta^{3}=-2 \beta$ has three distinct solutions. Thus, $\tilde{S}$ is defined by $\Psi_{1}=0$ or $\Psi_{\zeta}=0$ for a cubic root $\zeta$ of $-2 \beta$.

REMARK 2.9. Let $\tilde{S}_{\zeta}$ be a surface defined by $\Psi_{\zeta}$ for a cubic root $\zeta$ of $-2 \beta$. We set $S_{\zeta}=\tilde{S}_{\zeta} / G$. In the proof of Proposition 2.8, we saw that $S_{\zeta}$ has the singular fiber at $o$ which consists of four lines intersecting at the point $(o,(\zeta:-1: 1))$. If $\tilde{S}_{\zeta}$ has a singular point in the fiber at $o$, then it must be this point. Hence, we have the equation $\partial_{t} \Psi_{\zeta}(o,(\zeta:-1: 1))=0$. It is easy to see that $\partial_{t} \Psi_{\zeta}(o,(\zeta:-1: 1))=27 \zeta^{4}-18 \mu$. If $\lambda \notin\{0,1\}$, then there exists no common solution of equations $3 \zeta^{4}-2\left(3 \alpha^{2}-2(\lambda+1) \alpha+\lambda\right)=0, \zeta^{3}+2 \beta=0, \beta^{2}-\alpha(\alpha-$ 1) $(\alpha-\lambda)$ and (3). Thus, $\tilde{S}_{\zeta}$ have no singular points on the fibers of $\tilde{S}_{\zeta}$ at $o, o^{\prime}$ and $o^{\prime \prime}$.

3. Smoothness of the three surfaces. Let $\tilde{S}_{1}$ be the surface defined by $\Psi_{1}=0$ and $\tilde{S}_{\zeta}$ the surface defined by $\Psi_{\zeta}=0$ in Remark 2.9. Since $\zeta$ is a cubic root of $-2 \beta$, there are three choices for $\zeta$. We set $S_{1}=\tilde{S}_{1} / G$ and $S_{\zeta}=\tilde{S}_{\zeta} / G$. We already know that $S_{1}$ is the nonsingular surface in Example 2.2. In this section, we show that $S_{\zeta}$ is non-singular. It suffices to show that $\tilde{S}_{\zeta}$ is non-singular. We first show the following lemma. 
LeMma 3.1. Let $\tilde{S}$ be the surface defined by $\Psi=\sum_{i=1}^{5} a_{i} \Psi_{i}=0$ in $\boldsymbol{P}_{\tilde{E}}$. Then $\tilde{S}$ is reduced. Furthermore, $\tilde{S}$ is reducible if and only if $\Psi$ satisfies $a_{1}=a_{3}=a_{4}$ and $a_{5}=0$.

Proof. If $\tilde{S}$ is reducible or non-reduced, then we can write $\tilde{S}=T_{1}+T_{2}$ by non-zero effective divisors $T_{1}$ and $T_{2}$. We assume that $T_{i}$ are in $\left|m_{i} H+\tilde{p}^{*} D_{i}\right|$ with $D_{i} \in \operatorname{Div}(\tilde{E})$ and $\operatorname{deg} D_{i}=n_{i}$ for $i=1,2$, and we assume $m_{1} \leq m_{2}$. The complete linear system $\left|m H+\tilde{p}^{*} D\right|$ with $\operatorname{deg} D=n$ contains an effective member if and only if:

(a) $m>0, m+n>0$

(b) $m>0, m+n=0, \mathcal{O}_{\tilde{E}}(D) \cong \mathcal{O}_{\tilde{E}}\left(-i o-j o^{\prime}-k o^{\prime \prime}\right)(i+j+k=m, i, j, k \geq 0)$; or

(c) $m=0, n>0$.

In particular, we have $m_{i} \geq 0$ and $m_{i}+n_{i} \geq 0$. Since $\tilde{S} \in\left|4 \tilde{H}-\tilde{p}^{*}[o]\right|$, we have $m_{1}+m_{2}=4$, and $\mathcal{O}_{\tilde{E}}\left(D_{1}+D_{2}\right) \cong \mathcal{O}_{\tilde{E}}(-[o])$. Hence, we have $n_{1}+n_{2}=-3$. Since $n_{1} \leq 0$ implies $n_{2} \geq-3$, there are three possibilities, i.e., (i) $m_{1}=2, n_{1}<0$, (ii) $m_{1}=0$ and (iii) $m_{1}=1$.

(i) Since $\tilde{S}$ is $G$-invariant, $T_{o^{\prime}}^{*} T_{1}$ and $T_{o^{\prime \prime}}^{*} T_{1}$ are also components of $\tilde{S}$. If $T_{1}$ is not $G$ invariant, then we have $m_{1}=0,1$. Thus, $T_{1}$ is $G$-invariant, i.e., $T_{o^{\prime}}^{*} D_{1}$ is linearly equivalent to $D_{1}$. Hence, we have $n_{1} \equiv 0(\bmod 3)$. From $m_{1}+n_{1} \geq 0$, we obtain $-2 \leq n_{1}<0$. It contradicts the condition $n_{1} \equiv 0(\bmod 3)$.

(ii) Since $n_{1}>0, m_{2}=4, n_{2}=-n_{1}-3$ and $m_{2}+n_{2}=-n_{1}+1 \geq 0$, we have $n_{1}=1$ and $n_{2}=-4$. Since $T_{1}=\tilde{p}^{*} D_{1}$ is not $G$-invariant, $\tilde{p}^{*} T_{o^{\prime}}^{*} D_{1}$ and $\tilde{p}^{*} T_{o^{\prime \prime}}^{*} D_{1}$ are also components of $\tilde{S}$. Hence, the divisor $\tilde{S}-\tilde{p}^{*}\left(D_{1}+T_{o^{\prime}}^{*} D_{1}+T_{o^{\prime \prime}}^{*} D_{1}\right)$ which is linearly equivalent to $4 \tilde{H}-\tilde{p}^{*}[o]-\tilde{p}^{*}\left(D_{1}+T_{o^{\prime}}^{*} D_{1}+T_{o^{\prime \prime}}^{*} D_{1}\right)$ must be effective. However, $4 \tilde{H}-\tilde{p}^{*}[o]-\tilde{p}^{*}\left(D_{1}+\right.$ $\left.T_{o^{\prime}}^{*} D_{1}+T_{o^{\prime \prime}}^{*} D_{1}\right)$ satisfies none of the conditions (a), (b) and (c).

(iii) Since $n_{1} \geq-1, m_{2}=3, n_{2}=-n_{1}-3$ and $m_{2}+n_{2}=-n_{1} \geq 0$, we have $n_{1}=-1$ or 0 .

(iii $\left.1_{1}\right)$ The case $n_{1}=-1$. Since $T_{1}$ is an effective divisor, $D_{1}$ is linearly equivalent to $-o,-o^{\prime}$ or $-o^{\prime \prime}$. In this case, the components of $\tilde{S}$ are linearly equivalent to $\tilde{H}-\tilde{F}_{o}, \tilde{H}-$ $\tilde{F}_{O^{\prime}}, \tilde{H}-\tilde{F}_{o^{\prime \prime}}$ and $\tilde{H}$. It is easy to see that a $G$-invariant surface which is linearly equivalent to $\tilde{H}$ is defined by $Z_{0}+Z_{1}+Z_{2}=0$. Therefore, $\Psi$ is divisible by $Z_{0}, Z_{1}, Z_{2}$ and $Z_{0}+Z_{1}+Z_{2}$. It is easy to see $a_{1}=a_{3}=a_{4}=a_{5}=0$, i.e., $\Psi=\Psi_{2}$.

(iii 2$) \quad$ The case $n_{1}=0$. If $T_{1}$ is not $G$-invariant, then we have $\tilde{S}-\left(T_{1}+T_{o^{\prime}}^{*} T_{1}+T_{o^{\prime \prime}}^{*} T_{1}\right)$ is linearly equivalent to $\tilde{H}-\tilde{p}^{*}[o]$. However, $T_{1}$ is $G$-invariant since $\tilde{H}-\tilde{p}^{*}[o]$ satisfies none of (a), (b) and (c). So $T_{2}$ is also $G$-invariant. Similarly as in Lemma 1.4, we see that $f Z_{0}^{3}+g Z_{1}^{3}+h Z_{2}^{3}$ is a section of $H^{0}\left(\boldsymbol{P}_{\tilde{E}}, \mathcal{O}_{\boldsymbol{P}_{\tilde{E}}}\left(3 \tilde{H}-\tilde{p}^{*}[o]\right)\right)^{G}$. Thus, we have $\Psi=a_{1}\left(Z_{0}+\right.$ $\left.Z_{1}+Z_{2}\right)\left(f Z_{0}^{3}+g Z_{1}^{3}+h Z_{2}^{3}\right)$, i.e., $a_{1}=a_{3}=a_{4}$ and $a_{5}=0$.

By Lemma 3.1, $\tilde{S}_{\zeta}$ defined by $\Psi_{\zeta}$ is irreducible and reduced. Since $\Psi_{\zeta}=\Psi_{1}-12 \zeta^{2} \Psi_{2}+$ $4\left(\Psi_{3}+\Psi_{4}\right)-6 \zeta^{-2} \Psi_{5}, \tilde{S}_{\zeta}$ has a non-trivial automorphism of order two. Let $\gamma_{i}$ and $\Gamma$ be as in Remark 2.7. By Remark 2.7(iii), if $\tilde{S}$ has a singular fiber at a point in $\tilde{E} \backslash \Gamma$, then $\tilde{S}$ has five other singular fibers. If $\tilde{S}$ has a singular fiber at a point in $\Gamma$, then $\tilde{S}$ is two other singular fibers. 
In order to prove the smoothness of $\tilde{S}_{\zeta}$, we consider the fiber at a point in $\Gamma$. We already know that fibers of $\tilde{S}_{\zeta}$ at $o, o^{\prime}$ and $o^{\prime \prime}$ consist of four lines intersecting at one point and do not contain singularities of $\tilde{S}_{\zeta}$. For $P \in \Gamma \backslash\left\{o, o^{\prime}, o^{\prime \prime}\right\}$, the set $\left\{P, P^{\prime}, P^{\prime \prime},-P,-P^{\prime},-P^{\prime \prime}\right\}$ contains a point of order two. So we prove that all of the fibers of $\tilde{S}_{\zeta}$ at points with order two are non-singular in the following lemma. By this lemma, all the fibers at points in $\Gamma$ are non-singular.

LEMMA 3.2. Let $\tilde{S}_{\zeta}$ be the surface defined by $\Psi_{\zeta}=0$ in Remark 2.9 and $P \in \tilde{E}$ a point of order two. The fiber of $\tilde{S}_{\zeta}$ at $P$ is non-singular.

Proof. The order of $P \in \tilde{E}$ is one or two if and only if $P$ is zero of the rational function $g-h$. Let $\left(\tilde{S}_{\zeta}\right)_{P}$ be the fiber of $\tilde{S}_{\zeta}$ at $P$ and $\Psi_{\zeta}(P)$ the defining polynomial of $\left(\tilde{S}_{\zeta}\right)_{P}$. We denote $f(P)$ and $g(P)=h(P)$ simply by $f$ and $g$, respectively. Suppose that $\left(\tilde{S}_{\zeta}\right)_{P}$ have a singular point $Q$. Then $Q$ is a common zero of the following equations:

$$
\begin{aligned}
\partial_{Z_{0}} \Psi_{\zeta}(P)= & 4 f Z_{0}^{3}-12 \zeta^{2} Z_{1} Z_{2}\left(2 Z_{0}+Z_{1}+Z_{2}\right)+4 g\left(Z_{1}^{3}+Z_{2}^{3}\right) \\
& +12 f Z_{0}^{2}\left(Z_{1}+Z_{2}\right)-12 f g \zeta^{-2} Z_{0}\left(Z_{1}^{2}+Z_{2}^{2}\right)=0 \\
\partial_{Z_{1}} \Psi_{\zeta}(P)= & 4 f Z_{0}^{3}-12 \zeta^{2} Z_{0} Z_{2}\left(Z_{0}+2 Z_{1}+Z_{2}\right)+4 g\left(Z_{1}^{3}+Z_{2}^{3}\right) \\
& +12 g Z_{1}^{2}\left(Z_{0}+Z_{2}\right)-12 g \zeta^{-2} Z_{1}\left(f Z_{0}^{2}+g Z_{2}^{2}\right)=0 \\
\partial_{Z_{2}} \Psi_{\zeta}(P)= & 4 f Z_{0}^{3}-12 \zeta^{2} Z_{0} Z_{1}\left(Z_{0}+Z_{1}+2 Z_{2}\right)+4 g\left(Z_{1}^{3}+Z_{2}^{3}\right) \\
& +12 g Z_{2}^{2}\left(Z_{0}+Z_{1}\right)-12 g \zeta^{-2} Z_{2}\left(f Z_{0}^{2}+g Z_{1}^{2}\right)=0
\end{aligned}
$$

By taking the difference (44) - (45), we obtain the condition

$$
12 \zeta^{-2} g\left(\zeta^{2}+g\right)\left(Z_{1}-Z_{2}\right)\left(\zeta^{2} g^{-1} Z_{0}+Z_{1}\right)\left(\zeta^{2} g^{-1} Z_{0}+Z_{2}\right)=0 .
$$

Therefore, $Q$ is a solution of (i) $\zeta^{2}+g=0$, (ii) $Z_{1}=Z_{2}$, (iii) $\zeta^{2} g^{-1} Z_{0}+Z_{1}=0$ or (iv) $\zeta^{2} g^{-1} Z_{0}+Z_{2}=0$.

(i) The case $g=-\zeta^{2}$. Since $f g h=-\zeta^{6}$ by Remark 1.5, we obtain $f=g=h=$ $-\zeta^{2}$. However, as we saw in the proof of Proposition 2.8, $f(P)=g(P)=h(P)$ does not occur. Hence, we obtain $g \neq-\zeta^{2}$.

(ii) The case $Z_{1}=Z_{2}$. Since $(P,(1: 0: 0))$ is not contained in $\tilde{S}_{\zeta}$, we can write $Q=\left(Z_{0}: 1: 1\right)$. By substituting 1 for $Z_{1}$ and $Z_{2}$ in (43) and (44), we obtain the following equations:

$$
\begin{gathered}
4 f Z_{0}^{3}-24 \zeta^{2}\left(Z_{0}+1\right)+8 g+24 f Z_{0}^{2}-24 f g \zeta^{-2} Z_{0}=0 \\
4 f Z_{0}^{3}-12 \zeta^{2} Z_{0}\left(Z_{0}+3\right)+8 g+12 g\left(Z_{0}+1\right)-12 g \zeta^{-2}\left(f Z_{0}^{2}+g\right)=0 .
\end{gathered}
$$

The resultant of the left-hand sides of (46) and (47) with respect to $Z_{0}$ is $g^{-6}\left(g+\zeta^{2}\right)^{2}(-g+$ $\left.2 \zeta^{2}\right)^{3}$. Since $Q$ is a common solution of (46) and (47), we have $g^{-6}\left(g+\zeta^{2}\right)^{2}\left(-g+2 \zeta^{2}\right)^{3}=0$. So we have $g=h=2 \zeta^{2}$ and $f=-\zeta^{2} / 4=-g / 8$. We can show that there exists no point $P \in \tilde{E}$ which satisfies $-8 f(P)=g(P)=h(P)$ similarly as we proved that there exists no 
point $P \in \tilde{E}$ satisfying $f(P)=g(P)=h(P)$ in the proof of Proposition 2.8. Thus, we have $Z_{1} \neq Z_{2}$.

(iii) The case $\zeta^{2} g^{-1} Z_{0}+Z_{1}=0$. By replacing $Z_{0}$ of (43) and (44) with $-\zeta^{-2} g Z_{1}$, $Q$ is a solution of the following equations:

$$
\begin{aligned}
& 8 g Z_{1}^{3}+24 g Z_{1}^{2} Z_{2}-24 \zeta^{2} Z_{1}^{2} Z_{2}-24 \zeta^{2} Z_{1} Z_{2}^{2}+4 g Z_{2}^{3}-24 \zeta^{2} Z_{1}^{3}=0 \\
& 20 g Z_{1}^{3}-12 g^{2} \zeta^{-2} Z_{1}^{2} Z_{2}-12 g^{2} \zeta^{-2} Z_{1}^{3}-12 g^{2} \zeta^{-2} Z_{1} Z_{2}^{2}+36 g Z_{1}^{2} Z_{2} \\
& \quad+12 g Z_{1} Z_{2}^{2}+4 g Z_{2}^{3}=0 .
\end{aligned}
$$

Since the resultant of (48) and (49) with respect to $Z_{2}$ is zero, we obtain the condition $\left(g+\zeta^{2}\right)\left(-g+2 \zeta^{2}\right) Z_{1}=0$. If $Z_{1}=0$, then we have $Z_{0}=Z_{1}=Z_{2}=0$. Thus, we have $\zeta^{2} g^{-1} Z_{0}+Z_{1} \neq 0$.

(iv) We can show $\zeta^{2} g^{-1} Z_{0}+Z_{2} \neq 0$ in the same way. Since $g+\zeta^{2} \neq 0$ and $g-2 \zeta^{2} \neq$ 0 , (43), (44) and (45) have no common zero. So $\left(\tilde{S}_{\zeta}\right)_{P}$ is non-singular.

Next we show that $\tilde{S}_{\zeta}$ is normal.

Lemma 3.3. Let $\tilde{S}_{\zeta}$ be as in Lemma 3.2. Then $\tilde{S}_{\zeta}$ is normal.

PROOF. Since the algebraic surface $\tilde{S}_{\zeta}$ is defined by one equation $\Psi_{\zeta}=0$ in the nonsingular variety $\boldsymbol{P}_{\tilde{E}}$, it suffices to show that the codimension of the singular locus $\operatorname{Sing} \tilde{S}_{\zeta}$ is at least two. If there exists an irreducible component $D$ of $\operatorname{Sing} \tilde{S}_{\zeta}$ with $\operatorname{dim} D=1$, then $\tilde{\pi}(D)$ is either a point or an elliptic curve $\tilde{E}$. However, we know that $\tilde{S}_{\zeta}$ has non-singular fibers. So we can assume that $\tilde{\pi}(D)$ is a point $R \in \tilde{E}$. By Lemma 3.2, the order of $R$ is not two. Let $\left(\tilde{S}_{\zeta}\right)_{R}$ be the fiber of $\tilde{S}_{\zeta}$ at $R \in \tilde{E}$ and $\Psi_{\zeta}(R)$ the defining polynomial of $\left(\tilde{S}_{\zeta}\right)_{R}$. Then $\left(\tilde{S}_{\zeta}\right)_{R}$ contains a multiple line or a multiple conic.

(i) The case that $\left(\tilde{S}_{\zeta}\right)_{R}$ contains a line whose multiplicity is at least two. We denote $f=f(R), g=g(R)$ and $h=h(R)$, for simplicity. In this case, $\left(\tilde{S}_{\zeta}\right)_{R}$ is written as follows:

$$
\Psi_{\zeta}(R)=\left(b_{0} Z_{0}+b_{1} Z_{1}+b_{2} Z_{2}\right)^{2}\left(c_{0} Z_{0}^{2}+c_{1} Z_{0} Z_{1}+c_{2} Z_{0} Z_{2}+c_{3} Z_{1}^{2}+c_{4} Z_{1} Z_{2}+c_{5} Z_{2}^{2}\right) .
$$

By comparing this with the expression of $\Psi_{\zeta}(R)$ in Proposition 2.8, we obtain the following equations:

$$
\begin{gathered}
b_{0}^{2} c_{0}-f=0, \\
2 b_{0} b_{1} c_{0}+b_{0}^{2} c_{1}-4 f=0, \\
2 b_{0} b_{2} c_{0}+b_{0}^{2} c_{2}-4 f=0, \\
b_{1}^{2} c_{0}+2 b_{0} b_{1} c_{1}+b_{0}^{2} c_{3}+6 \zeta^{-2} f g=0, \\
2 b_{1} b_{2} c_{0}+2 b_{0} b_{2} c_{1}+2 b_{0} b_{1} c_{2}+b_{0}^{2} c_{4}+12 \zeta^{2}=0, \\
b_{2}^{2} c_{0}+2 b_{0} b_{2} c_{2}+b_{0}^{2} c_{5}+6 \zeta^{-2} f h=0, \\
b_{1}^{2} c_{1}+2 b_{0} b_{1} c_{3}-4 g=0, \\
2 b_{1} b_{2} c_{1}+b_{1}^{2} c_{2}+2 b_{0} b_{2} c_{3}+2 b_{0} b_{1} c_{4}+12 \zeta^{2}=0,
\end{gathered}
$$




$$
\begin{gathered}
b_{2}^{2} c_{1}+2 b_{1} b_{2} c_{2}+2 b_{0} b_{2} c_{4}+2 b_{0} b_{1} c_{5}+12 \zeta^{2}=0, \\
b_{2}^{2} c_{2}+2 b_{0} b_{2} c_{5}-4 h=0, \\
b_{1}^{2} c_{3}-g=0, \\
2 b_{1} b_{2} c_{3}+b_{1}^{2} c_{4}-4 g=0, \\
b_{2}^{2} c_{4}+2 b_{1} b_{2} c_{5}-4 h=0, \\
b_{2}^{2} c_{5}-h=0 .
\end{gathered}
$$

Since $f \neq 0$, we can assume $b_{0}=1$. By (50)-(55), we see that $c_{0}, \ldots, c_{5}$ are polynomials in $b_{1}, b_{2}$. Furthermore, by (56)-(63), we obtain the following conditions on $b_{1}$ and $b_{2}$.

$$
\begin{gathered}
-12 b_{1}^{2} f+4 b_{1}^{3} f-4 g-12 b_{1} f g \zeta^{-2}=0, \\
-12 b_{1}^{2} f-24 b_{1} b_{2} f+12 b_{1}^{2} b_{2} f-12 b_{2} f g \zeta^{-2}+12 \zeta^{2}-24 b_{1} \zeta^{2}=0, \\
-24 b_{1} b_{2} f-12 b_{2}^{2} f+12 b_{1} b_{2}^{2} f-12 b_{1} f h \zeta^{-2}+12 \zeta^{2}-24 b_{2} \zeta^{2}=0, \\
-12 b_{2}^{2} f+4 b_{2}^{3} f-4 h-12 b_{2} f h \zeta^{-2}=0, \\
-8 b_{1}^{3} f+3 b_{1}^{4} f-g-6 b_{1}^{2} f g \zeta^{-2}=0, \\
-8 b_{1}^{3} f-24 b_{1}^{2} b_{2} f+12 b_{1}^{3} b_{2} f-4 g-12 b_{1} b_{2} f g \zeta^{-2}-12 b_{1}^{2} \zeta^{2}=0, \\
-24 b_{1} b_{2}^{2} f-8 b_{2}^{3} f+12 b_{1} b_{2}^{3} f-4 h-12 b_{1} b_{2} f h \zeta^{-2}-12 b_{2}^{2} \zeta^{2}=0, \\
-8 b_{2}^{3} f+3 b_{2}^{4} f-h-6 b_{2}^{2} f h \zeta^{-2}=0 .
\end{gathered}
$$

By taking the differences $b_{1} \times(65)-(69)$ and $b_{2} \times(66)-(70)$, we have

$$
\begin{aligned}
& -4 b_{1}^{3} f+4 g+12 b_{1} \zeta^{2}-12 b_{1}^{2} \zeta^{2}=0, \\
& -4 b_{2}^{3} f+4 h+12 b_{2} \zeta^{2}-12 b_{2}^{2} \zeta^{2}=0 .
\end{aligned}
$$

The resultant of (64) $+c \times(68)(c \in \boldsymbol{C})$ and (72) with respect to $b_{1}$ is written as polynomial in $c$ and vanishes for any $c \in \boldsymbol{C}$. Hence all coefficients of this polynomial are zero.

$$
\begin{gathered}
f^{2} g^{2}+2 f^{2} g h+3 f g^{2} h+2 f g h^{2}+g^{2} h^{2}-6 f g h \zeta^{2}-6 f g \zeta^{4} \\
-3 f h \zeta^{4}-6 g h \zeta^{4}=0, \\
-3 f^{2} g^{2}-8 f^{2} g h-10 f g^{2} h-6 f g h^{2}-3 g^{2} h^{2}-f^{2} h \zeta^{2}+16 f g h \zeta^{2}+19 f g \zeta^{4} \\
+8 f h \zeta^{4}+18 g h \zeta^{4}=0 .
\end{gathered}
$$

Similarly, by taking the resultant of $(67)+c \times(71)(c \in \boldsymbol{C})$ and (73) with respect to $b_{2}$, we obtain the following equations:

$$
\begin{aligned}
2 f^{2} g h & +2 f g^{2} h+f^{2} h^{2}+3 f g h^{2}+g^{2} h^{2}-6 f g h \zeta^{2}-3 f g \zeta^{4} \\
- & 6 f h \zeta^{4}-6 g h \zeta^{4}=0
\end{aligned}
$$




$$
\begin{aligned}
& -8 f^{2} g h-6 f g^{2} h-3 f^{2} h^{2}-10 f g h^{2}-3 g^{2} h^{2}-f^{2} g \zeta^{2}+16 f g h \zeta^{2}+8 f g \zeta^{4} \\
& \quad+19 f h \zeta^{4}+18 g h \zeta^{4}=0 .
\end{aligned}
$$

By computing the differences (74) - (76) and (75) - (77), we have

$$
\begin{gathered}
-f(g-h)\left(f g+f h+g h-3 \zeta^{4}\right)=0, \\
f(g-h)\left(3 f g+3 f h+4 g h-f \zeta^{2}-11 \zeta^{4}\right)=0 .
\end{gathered}
$$

Because the order of $R$ is not two, $g-h \neq 0$ and we have

$$
\begin{gathered}
f g+f h+g h-3 \zeta^{4}=0, \\
3 f g+3 f h+4 g h-f \zeta^{2}-11 \zeta^{4}=0 .
\end{gathered}
$$

However, if these equations hold, then we have $g=h$ by an easy calculation because we have $f g h=-4 \beta^{2}=-\zeta^{6}$. Hence, this case does not occur.

(ii) The case that $\left(\tilde{S}_{\zeta}\right)_{R}$ contains a conic whose multiplicity is two. In this case, $\Psi_{\zeta}(R)$ can be written as

$$
\Psi_{\zeta}(R)=\left(b_{0} Z_{0}^{2}+b_{1} Z_{0} Z_{1}+b_{2} Z_{0} Z_{2}+b_{3} Z_{1}^{2}+b_{4} Z_{1} Z_{2}+b_{5} Z_{2}^{2}\right)^{2} .
$$

By comparing coefficients of $\Psi_{\zeta}(R)$, we obtain the following equations:

$$
\begin{gathered}
b_{0}^{2}-f=0, \\
2 b_{0} b_{1}-4 f=0, \\
2 b_{0} b_{2}-4 f=0, \\
b_{1}^{2}+2 b_{0} b_{3}+6 \zeta^{-2} f g=0, \\
2 b_{1} b_{2}+2 b_{0} b_{4}+12 \zeta^{2}=0, \\
b_{2}^{2}+2 b_{0} b_{5}+6 \zeta^{-2} f h=0, \\
2 b_{1} b_{3}-4 g=0, \\
2 b_{2} b_{5}-4 h=0 .
\end{gathered}
$$

By computing (78)-(82), we obtain the conditions

$$
\begin{gathered}
b_{1}=2 b_{0}, \quad b_{2}=2 b_{0}, \quad b_{3}=-2 b_{0}-3 \zeta^{-2} g b_{0}, \\
b_{4}=\left(4+6 \zeta^{2} / f\right) b_{0}, \quad b_{5}=-2 b_{0}-3 \zeta^{-2} h b_{0} .
\end{gathered}
$$

By (84) and (85), we obtain the following equalities:

$$
\begin{aligned}
& \left(-2-3 \zeta^{-2} g\right) b_{0}^{2}-g=0, \\
& \left(-2-3 \zeta^{-2} h\right) b_{0}^{2}-h=0 .
\end{aligned}
$$

Here we get $g=h$ which contradicts our assumption. Thus, the lemma is proved.

By using Lemma 3.3, we will show that $\tilde{S}_{\zeta}$ is non-singular.

LEMMA 3.4. The surface $\tilde{S}_{\zeta}$ defined for a cubic root $\zeta$ of $-2 \beta$ in Remark 2.9 is nonsingular. 
PROOF. Since $\tilde{S}_{\zeta}$ is normal, singular points of $\tilde{S}_{\zeta}$ are isolated. Let $v: S_{\zeta}^{*} \rightarrow \tilde{S}_{\zeta}$ be the minimal resolution. Let $p_{g}\left(\tilde{S}_{\zeta}, P\right)$ be the geometric genus of $\left(\tilde{S}_{\zeta}, P\right)$. Then, by the Leray spectral sequence, we have

$$
\chi\left(\mathcal{O}_{\tilde{S}_{\zeta}}\right)-\chi\left(\mathcal{O}_{S_{\zeta}^{*}}\right)=\sum_{P \in \operatorname{Sing}} p_{\tilde{S}_{\zeta}}\left(\tilde{S}_{\zeta}, P\right) .
$$

If $\tilde{S}_{\zeta}$ has a singular point, then $\tilde{S}_{\zeta}$ has two other singular points since the cyclic group of order three acts on it freely. Therefore, the right-hand side of (88) is a positive multiple of three.

We have $\kappa\left(S_{\zeta}^{*}\right) \geq 1$, since $S_{\zeta}^{*}$ has a fibration of curves of genus three over an elliptic curve. In particular, $\chi\left(\mathcal{O}_{S_{\zeta}^{*}}\right) \geq 0$. By [9, Proposition 2.3], we know $\chi\left(\mathcal{O}_{\tilde{S}_{\zeta}}\right)=3$. Hence, the left-hand side of (88) is at most three. Therefore, if there exist singular points which are not rational double points on $\tilde{S}_{\zeta}$, then these singular points are minimal elliptic singular points and the number of them is three. By Remark 2.7(iv), if $\tilde{S}_{\zeta}$ has a singular point in the fiber at $P \in \tilde{E} \backslash \Gamma$, then $\tilde{S}_{\zeta}$ has six singular points. Since there exist only three minimal elliptic singular points on $\tilde{S}_{\zeta}$, these points are mapped into $\Gamma$ and one of these singular points is contained in a fiber at a point with order two.

However, we already know that a fiber at a point with order two is non-singular. Thus, $\tilde{S}_{\zeta}$ has at worst rational double points. Because the sum of the Euler contributions is 27, $\tilde{S}_{\zeta}$ does not have other singular fibers and rational double points. Hence, we see that $\tilde{S}_{\zeta}$ is non-singular.

We showed that $\tilde{S}_{1}$ and $\tilde{S}_{\zeta}$ are non-singular in Example 2.2 and Lemma 3.4. We know that, for a given elliptic curve $E$, there exist almost four isomorphism classes of the CataneseCiliberto surfaces of type I with $E \cong \operatorname{Alb}(S)$.

4. Isomorphic classes of surfaces. In this section, we consider the number of isomorphism classes of Catanese-Ciliberto surfaces $S$ of type I with $\operatorname{Alb}(S) \cong E$ for a given $E$. In order to count the number, we use the defining equations of these surfaces in $\boldsymbol{P}_{E}$.

4.1. A transition function of an indecomposable bundle. Let $E$ be an elliptic curve and $V$ an indecomposable bundle of rank three with det $V \cong \mathcal{O}_{E}(o)$. In order to describe the defining equations of canonical models of Catanese-Ciliberto surfaces in $\boldsymbol{P}_{E}(V)$, we describe a transition function system of $V$.

We embed the elliptic curve $E$ in $\boldsymbol{P}^{2}$ so that it satisfies the equality $Y^{2} Z=X(X-$ $Z)(X-\lambda Z)$ for $\lambda \in C \backslash\{0,1\}$ and $o=(0: 1: 0)$. Now we recall the following lemma which we need.

LEMMA 4.1 (Atiyah [1, Lemma 16]). Let $r$ and $d$ be positive integers. Let $V^{\prime}$ be an indecomposable bundle of rank $r$ and degree $d$ over $E$. Then there exists an indecomposable bundle of rank $r+d$ and degree $d$ over $E$, unique up to an isomorphisms, given by an extension

$$
0 \rightarrow \mathcal{O}_{E}^{\oplus s} \rightarrow V \rightarrow V^{\prime} \rightarrow 0
$$


By the above lemma, there exists a unique indecomposable bundle $V_{2,1}$ of rank two and degree one over $E$ satisfying the extension $0 \rightarrow \mathcal{O}_{E} \rightarrow V_{2,1} \rightarrow \mathcal{O}_{E}(o) \rightarrow 0$. Moreover, we see that there exists a unique indecomposable bundle $V$ of rank three and degree one over $E$ satisfying the extension $0 \rightarrow \mathcal{O}_{E} \rightarrow V \rightarrow V_{2,1} \rightarrow 0$. Note that the determinant line bundle of the above vector bundle $V$ is equal to $\mathcal{O}_{E}(o)$.

Let $U_{Y}=\left\{\left(q_{0}: q_{1}: q_{2}\right) \in E \mid q_{1} \neq 0\right\}, U_{Z}=\left\{\left(q_{0}: q_{1}: q_{2}\right) \in E \mid q_{2} \neq 0\right\}$ and $U_{Y Z}=U_{Y} \cap U_{Z}$. Let $r_{1}, r_{2}$ and $r_{3}$ be the points in $E$ of order two. Then $U_{Y}=E \backslash\left\{r_{1}, r_{2}, r_{3}\right\}$ and $U_{Z}=E \backslash\{o\}$. We describe a transition matrix of $V$ at $U_{Y Z}$ for bases $\left\{Y_{0}, Y_{1}, Y_{2}\right\}$ of $\left.V\right|_{U_{Y}}$ and $\left\{Z_{0}, Z_{1}, Z_{2}\right\}$ of $\left.V\right|_{U_{Z}}$ as follows. Note that $Z_{i}$ in this section are not equal to those in Section 3. Set $t=X / Y$. Then $t$ is regular on $U_{Y}$ and has zero of order one at $o$. So a transition function of $\mathcal{O}_{E}(o)$ at $U_{Y Z}$ is given by $\left\{t^{-1}\right\}$. Since $V_{2,1}$ is given by a non-zero element of $H^{1}\left(E, \mathcal{H o m}\left(\mathcal{O}_{E}(o), \mathcal{O}_{E}\right)\right)$, a transition matrix of $V_{2,1}$ at $U_{Y Z}$ is given by

$$
\left(\begin{array}{cc}
1 & 0 \\
t^{-2} & t^{-1}
\end{array}\right)
$$

Furthermore, since $V$ is given by a non-zero element of $H^{1}\left(E, \mathcal{H o m}\left(V_{2,1}, \mathcal{O}_{E}\right)\right)$, a transition matrix of $V$ at $U_{Y Z}$ is given by

$$
\left(\begin{array}{l}
Y_{0} \\
Y_{1} \\
Y_{2}
\end{array}\right)=\left(\begin{array}{ccc}
1 & 0 & 0 \\
t^{-1} & 1 & 0 \\
0 & t^{-2} & t^{-1}
\end{array}\right)\left(\begin{array}{l}
Z_{0} \\
Z_{1} \\
Z_{2}
\end{array}\right) .
$$

4.2. The defining equations in $\boldsymbol{P}_{E}(\boldsymbol{V})$. In this section, we give defining polynomials of Catanese-Ciliberto surfaces which are elements of $H^{0}\left(\boldsymbol{P}_{E}(V), \mathcal{O}_{\boldsymbol{P}_{E}(V)}\left(4 H-F_{o}\right)\right)$. Since we have $H^{0}\left(\boldsymbol{P}_{E}(V), \mathcal{O}_{\boldsymbol{P}_{E}(V)}\left(4 H-F_{o}\right)\right) \cong H^{0}\left(E, \operatorname{Sym}^{4} V \otimes \mathcal{O}_{E}(-o)\right)$, it suffices to give elements of $H^{0}\left(E, \operatorname{Sym}^{4} V \otimes \mathcal{O}_{E}(-o)\right)$. By the previous section, we have $\left.V\right|_{U_{Y}}=$ $\bigoplus_{i=0,1,2} \mathcal{O}_{U_{Y}} Y_{i}$ and $\left.V\right|_{U_{Z}}=\bigoplus_{i=0,1,2} \mathcal{O}_{U_{Z}} Z_{i}$ with the relation (89). Then we have

$$
\begin{gathered}
H^{0}\left(U_{Y}, \operatorname{Sym}^{4} V \otimes \mathcal{O}_{E}(-o)\right)=\bigoplus_{i+j+k=4} \mathcal{O}_{E}(-o)\left(U_{Y}\right) Y_{0}^{i} Y_{1}^{j} Y_{2}^{k}, \\
H^{0}\left(U_{Z}, \operatorname{Sym}^{4} V \otimes \mathcal{O}_{E}(-o)\right)=\bigoplus_{i+j+k=4} \mathcal{O}_{E}\left(U_{Z}\right) Z_{0}^{i} Z_{1}^{j} Z_{2}^{k} .
\end{gathered}
$$

The sections $Y_{0}^{i} Y_{1}^{j} Y_{2}^{k}$ and $Z_{0}^{i} Z_{1}^{j} Z_{2}^{k}$ satisfy the relation on $U_{Y} \cap U_{Z}$ as follows:

$$
Y_{0}^{i} Y_{1}^{j} Y_{2}^{k}=\left(Z_{0}\right)^{i}\left(t^{-2} Z_{0}+Z_{1}\right)^{j}\left(t^{-2} Z_{1}+t^{-1} Z_{2}\right)^{k} .
$$

We give an explicit basis of the vector space $H^{0}\left(\boldsymbol{P}_{E}(V), \mathcal{O}_{\boldsymbol{P}_{E}(V)}\left(4 H-F_{o}\right)\right)$ in the following lemma.

Lemma 4.2. Let $E, \lambda, U_{Z}, Z_{0}, Z_{1}$ and $Z_{2}$ be as above. Set $x=X / Z$ and $y=Y / Z$. Then $H^{0}\left(\boldsymbol{P}_{E}(V), \mathcal{O}_{\boldsymbol{P}_{E}(V)}\left(4 H-F_{o}\right)\right)$ has a basis $\left\{F_{1}, F_{2}, F_{3}, F_{4}, F_{5}\right\}$ defined by

$$
\begin{aligned}
F_{1}= & 5 \lambda^{2} Z_{0}^{4}+8 \lambda y Z_{0}^{3} Z_{1}+8 \lambda x Z_{0}^{3} Z_{2}+\left(4(\lambda+1) x^{2}-2\left(2 \lambda^{2}+\lambda+2\right) x-2 \lambda(\lambda+1)\right) Z_{0}^{2} Z_{1}^{2} \\
& +8(\lambda+1) y Z_{0}^{2} Z_{1} Z_{2}+(4(\lambda+1) x-6 \lambda) Z_{0}^{2} Z_{2}^{2}+\left(-3 x^{2}+2(\lambda+1) x+(\lambda-1)^{2}\right) Z_{1}^{4} \\
& -8 y Z_{1}^{3} Z_{2}+(-6 x+2(\lambda+1)) Z_{1}^{2} Z_{2}^{2}+Z_{2}^{4},
\end{aligned}
$$




$$
\begin{aligned}
F_{2}= & \left(-3 \lambda^{2} x+4 \lambda^{2}(\lambda+1)\right) Z_{0}^{4}+8 \lambda^{2} Z_{0}^{3} Z_{2}+\left(2 \lambda x^{2}-2 \lambda(\lambda+1) x-4 \lambda^{2}\right) Z_{0}^{2} Z_{1}^{2} \\
& +4 \lambda y Z_{0}^{2} Z_{1} Z_{2}+2 \lambda x Z_{0}^{2} Z_{2}^{2}+\left(x^{3}-2(\lambda+1) x^{2}+(\lambda+1)^{2} x\right) Z_{1}^{4} \\
& +(4 y(x-1)-4 \lambda y) Z_{1}^{3} Z_{2}+\left(6 x^{2}-6(\lambda+1) x+4 \lambda\right) Z_{1}^{2} Z_{2}^{2}+4 y Z_{1} Z_{2}^{3}+x Z_{2}^{4}, \\
F_{3}= & -\lambda x Z_{0}^{4}-2(\lambda+1) y Z_{0}^{3} Z_{1}+(-2(\lambda+1) x+3 \lambda) Z_{0}^{3} Z_{2} \\
& +\left(-3 x^{2}+(\lambda+1) x+2\left(\lambda^{2}-\lambda+1\right)\right) Z_{0}^{2} Z_{1}^{2}-6 y Z_{0}^{2} Z_{1} Z_{2}+(-3 x+2(\lambda+1)) Z_{0}^{2} Z_{2}^{2} \\
& -2 y Z_{0} Z_{1}^{3}+(-3 x+(\lambda+1)) Z_{0} Z_{1}^{2} Z_{2}+Z_{0} Z_{2}^{3}, \\
F_{4}= & -\lambda^{2} Z_{0}^{4}-\lambda y Z_{0}^{3} Z_{1}-\lambda x Z_{0}^{3} Z_{2}+(-\lambda x+\lambda(\lambda+1)) Z_{0}^{2} Z_{1}^{2}+\lambda Z_{0}^{2} Z_{2}^{2} \\
& +(x y-(\lambda+1) y) Z_{0} Z_{1}^{3}+\left(3 x^{2}-3(\lambda+1) x+2 \lambda\right) Z_{0} Z_{1}^{2} Z_{2}+3 y Z_{0} Z_{1} Z_{2}^{2}+x Z_{0} Z_{2}^{3}, \\
F_{5}= & -\lambda^{2} y Z_{0}^{4}+\left(-4 \lambda^{2} x+2 \lambda^{2}(\lambda+1)\right) Z_{0}^{3} Z_{1}+6 \lambda y(x-(\lambda+1)) Z_{0}^{2} Z_{1}^{2} \\
& +\left(4(\lambda+1) x^{3}-4\left(2 \lambda^{2}+\lambda+2\right) x^{2}+2\left(2 \lambda^{3}+\lambda^{2}+\lambda+2\right) x-2(\lambda-1)^{2} \lambda\right) Z_{0} Z_{1}^{3} \\
& +12 \lambda(x-\lambda)(x-1) Z_{0}^{2} Z_{1} Z_{2}+6 \lambda y Z_{0}^{2} Z_{2}^{2}+12 y\left((\lambda+1) x-\left(\lambda^{2}+1\right)\right) Z_{0} Z_{1}^{2} Z_{2} \\
& +\left(12(\lambda+1) x^{2}-12\left(\lambda^{2}+\lambda+1\right) x+6 \lambda(\lambda+1)\right) Z_{0} Z_{1} Z_{2}^{2}+4(\lambda+1) y Z_{0} Z_{2}^{3} \\
& +y\left(3 x^{2}-2(\lambda+1) x-(\lambda-1)^{2}\right) Z_{1}^{4}+12\left(x^{3}-(\lambda+1) x^{2}+\lambda x\right) Z_{1}^{3} Z_{2} \\
& +(18 x y-6(\lambda+1) y) Z_{1}^{2} Z_{2}^{2}+\left(12 x^{2}-8(\lambda+1) x+4 \lambda\right) Z_{1} Z_{2}^{3}+3 y Z_{2}^{4} .
\end{aligned}
$$

Proof. Let $\gamma$ be an element of $H^{0}\left(E, \operatorname{Sym}^{4} V \otimes O_{E}(-o)\right)$. Then $\left.\gamma\right|_{U_{Y}}$ and $\left.\gamma\right|_{U_{Z}}$ are written as

$$
\begin{aligned}
\left.\gamma\right|_{U_{Y}}= & \alpha_{400} Y_{0}^{4}+\alpha_{310} Y_{0}^{3} Y_{1}+\alpha_{301} Y_{0}^{3} Y_{2}+\alpha_{220} Y_{0}^{2} Y_{1}^{2}+\alpha_{211} Y_{0}^{2} Y_{1} Y_{2} \\
& +\alpha_{202} Y_{0}^{2} Y_{2}^{2}+\alpha_{130} Y_{0} Y_{1}^{3}+\alpha_{121} Y_{0} Y_{1}^{2} Y_{2}+\alpha_{112} Y_{0} Y_{1} Y_{2}^{2}+\alpha_{103} Y_{0} Y_{2}^{3} \\
& +\alpha_{040} Y_{1}^{4}+\alpha_{031} Y_{1}^{3} Y_{2}+\alpha_{022} Y_{1}^{2} Y_{2}^{2}+\alpha_{013} Y_{1} Y_{2}^{3}+\alpha_{004} Y_{2}^{4}, \\
\left.\gamma\right|_{U_{Z}}= & \beta_{400} Z_{0}^{4}+\beta_{310} Z_{0}^{3} Z_{1}+\beta_{301} Z_{0}^{3} Z_{2}+\beta_{220} Z_{0}^{2} Z_{1}^{2}+\beta_{211} Z_{0}^{2} Z_{1} Z_{2} \\
& +\beta_{202} Z_{0}^{2} Z_{2}^{2}+\beta_{130} Z_{0} Z_{1}^{3}+\beta_{121} Z_{0} Z_{1}^{2} Z_{2}+\beta_{112} Z_{0} Z_{1} Z_{2}^{2}+\beta_{103} Z_{0} Z_{2}^{3} \\
& +\beta_{040} Z_{1}^{4}+\beta_{031} Z_{1}^{3} Z_{2}+\beta_{022} Z_{1}^{2} Z_{2}^{2}+\beta_{013} Z_{1} Z_{2}^{3}+\beta_{004} Z_{2}^{4},
\end{aligned}
$$

where $\alpha_{i j k}$ and $\beta_{i j k}$ are in $\mathcal{O}_{E}(-o)\left(U_{Y}\right)$ and $\mathcal{O}_{E}\left(U_{Z}\right)$, respectively.

Since $\left.\left(\left.\gamma\right|_{U_{Y}}\right)\right|_{U_{Y} \cap U_{Z}}=\left.\left(\left.\gamma\right|_{U_{Z}}\right)\right|_{U_{Y} \cap U_{Z}}$, we have the following relations of the coefficients.
(90) $\quad \alpha_{004}=t^{4} \beta_{004}$,
(91) $\alpha_{013}=t^{3} \beta_{013}-4 t^{2} \beta_{004}$,
(92) $\alpha_{022}=t^{2} \beta_{022}-3 t \beta_{013}+6 \beta_{004}$,
(93) $\alpha_{031}=t \beta_{031}-2 \beta_{022}+3 t^{-1} \beta_{013}-4 t^{-2} \beta_{004}$,
(94) $\alpha_{040}=\beta_{040}-t^{-1} \beta_{031}+t^{-2} \beta_{022}-t^{-3} \beta_{013}+t^{-4} \beta_{004}$, 
(95) $\alpha_{103}=t^{3} \beta_{103}-t^{2} \beta_{013}+4 t \beta_{004}$,

(96) $\alpha_{112}=t^{2} \beta_{112}-3 t \beta_{103}-2 t \beta_{022}+6 \beta_{013}-12 t^{-1} \beta_{004}$,

(97) $\alpha_{121}=t \beta_{121}-2 \beta_{112}+3 t^{-1} \beta_{103}-3 \beta_{031}+6 t^{-1} \beta_{022}-9 t^{-2} \beta_{013}+12 t^{-3} \beta_{004}$, $\alpha_{130}=\beta_{130}-t^{-1} \beta_{121}+t^{-2} \beta_{112}-t^{-3} \beta_{103}-4 t^{-1} \beta_{040}+4 t^{-2} \beta_{031}-4 t^{-3} \beta_{022}$ $+4 t^{-4} \beta_{013}-4 t^{-5} \beta_{004}$,

(99) $\alpha_{202}=t^{2} \beta_{202}-t \beta_{130}+3 \beta_{103}+\beta_{022}-3 t^{-1} \beta_{013}+6 t^{-2} \beta_{004}$,

$$
\begin{aligned}
\alpha_{211}= & t \beta_{211}-2 \beta_{202}-2 \beta_{121}+4 t^{-1} \beta_{112}-6 t^{-2} \beta_{103}+3 t^{-1} \beta_{031}-6 t^{-2} \beta_{022} \\
& +9 t^{-3} \beta_{013}-12 t^{-4} \beta_{004}, \\
\alpha_{220}= & \beta_{220}-t^{-1} \beta_{211}+t \beta^{-2} \beta_{202}-3 t^{-1} \beta_{103}+3 t^{-2} \beta_{121}-3 t^{-3} \beta_{112} \\
& +3 t^{-4} \beta_{103}+6 t^{-2} \beta_{040}-6 t^{-3} \beta_{031}+6 t^{-4} \beta_{022}-6 t^{-5} \beta_{013}+6 t^{-6} \beta_{004}, \\
\alpha_{301}= & t \beta_{301}-\beta_{211}+2 t^{-1} \beta_{202}+t^{-1} \beta_{121}-2 t^{-2} \beta_{112}+3 t^{-3} \beta_{103}-t^{-2} \beta_{031} \\
& +2 t^{-3} \beta_{022}-3 t^{-4} \beta_{013}+4 t^{-5} \beta_{004}, \\
\alpha_{310}= & \beta_{310}-t^{-1} \beta_{301}-2 t^{-1} \beta_{220}+2 t^{-2} \beta_{211}-2 t^{-3} \beta_{202}+3 t^{-2} \beta_{130} \\
& -3 t^{-3} \beta_{121}+3 t^{-4} \beta_{112}-3 t^{-3} \beta_{103}-4 t^{-3} \beta_{040}+4 t^{-4} \beta_{031} \\
& -4 t^{-5} \beta_{022}+4 t^{-6} \beta_{013}-4 t^{-7} \beta_{004}, \\
\alpha_{400}= & \beta_{400}-t^{-1} \beta_{310}+t^{-2} \beta_{301}+t^{-2} \beta_{220}-t^{-3} \beta_{211}+t^{-4} \beta_{202}-t^{-3} \beta_{130} \\
& +t^{-4} \beta_{121}-t^{-5} \beta_{112}+t^{-6} \beta_{103}+t^{-4} \beta_{040}-t^{-5} \beta_{031}+t^{-6} \beta_{022} \\
& -t^{-7} \beta_{013}+t^{-8} \beta_{004} .
\end{aligned}
$$

Let $v_{o}$ be the valuation of the local ring $\mathcal{O}_{E, o}$. Since $\alpha_{i j k}$ is in $\mathcal{O}_{E}(-o)\left(U_{Y}\right)$, we obtain $v_{o}\left(\alpha_{i j k}\right) \geq 1$. Hence, by (90) and $v_{o}(t)=1$, we have $v_{o}\left(\beta_{004}\right) \geq-3$, i.e., $\beta_{004} \in$ $H^{0}\left(E, \mathcal{O}_{E}(3 o)\right)$. By $(91)$, we have

$$
v_{o}\left(t^{3} \beta_{013}\right) \geq \min \left\{v_{o}\left(\alpha_{013}\right), v_{o}\left(t^{2}\right)+v_{o}\left(\beta_{004}\right)\right\} \geq-1 .
$$

Thus, we obtain $v_{o}\left(\beta_{013}\right) \geq-4$, i.e., $\beta_{013} \in H^{0}\left(E, \mathcal{O}_{E}(4 o)\right)$. Similarly, we obtain $\beta_{i j k} \in$ $H^{0}\left(E, \mathcal{O}_{E}((3+j) o)\right)$. Since $\left\{1, x, y, x^{2}, x y, x^{3}, x^{2} y\right\}$ is a $C$-basis of $H^{0}\left(E, \mathcal{O}_{E}(7 o)\right), \beta_{i j k}$ can be written as follows:

$$
\begin{aligned}
& \beta_{004}=a_{0}+a_{1} x+a_{2} y, \\
& \beta_{013}=b_{0}+b_{1} x+b_{2} y+b_{3} x^{2}, \\
& \beta_{022}=c_{0}+c_{1} x+c_{2} y+c_{3} x^{2}+c_{4} x y, \\
& \beta_{031}=d_{0}+d_{1} x+d_{2} y+d_{3} x^{2}+d_{4} x y+d_{5} x^{3}, \\
& \beta_{040}=e_{0}+e_{1} x+e_{2} y+e_{3} x^{2}+e_{4} x y+e_{5} x^{3}+e_{6} x^{2} y,
\end{aligned}
$$




$$
\begin{aligned}
& \beta_{103}=f_{0}+f_{1} x+f_{2} y, \\
& \beta_{112}=g_{0}+g_{1} x+g_{2} y+g_{3} x^{2}, \\
& \beta_{121}=h_{0}+h_{1} x+h_{2} y+h_{3} x^{2}+h_{4} x y, \\
& \beta_{130}=i_{0}+i_{1} x+i_{2} y+i_{3} x^{2}+i_{4} x y+i_{5} x^{3}, \\
& \beta_{202}=j_{0}+j_{1} x+j_{2} y, \\
& \beta_{211}=k_{0}+k_{1} x+k_{2} y+k_{3} x^{2}, \\
& \beta_{220}=l_{0}+l_{1} x+l_{2} y+l_{3} x^{2}+l_{4} x y, \\
& \beta_{301}=m_{0}+m_{1} x+m_{2} y, \\
& \beta_{310}=n_{0}+n_{1} x+n_{2} y+n_{3} x^{2}, \\
& \beta_{400}=p_{0}+p_{1} x+p_{2} y,
\end{aligned}
$$

where $a_{0}, a_{1}, \ldots, p_{1}, p_{2}$ are in $\boldsymbol{C}$. Since $\alpha_{i j k}$ has zero at $o$, by these equations and (90)-(104), we obtain relations of the complex numbers $a_{0}, a_{1}, \ldots, p_{1}, p_{2}$. By the equality (91), we have

$$
\alpha_{013}=t^{-1}\left(b_{3} u^{2}-4 a_{1} u\right)+\left(b_{2}-4 a_{1}\right) u+(\text { higher term }) .
$$

Since $v_{0}\left(\alpha_{013}\right) \geq 1$, we obtain conditions $b_{3}=4 a_{2}$ and $b_{2}=4 a_{1}$. By these equalities and (92), we have

$$
\begin{aligned}
\alpha_{022}= & t^{-3}\left(\left(c_{4}-12 a_{2}\right) u^{2}+6 a_{2} u\right)+t^{-2}\left(c_{3} u^{2}-6 a_{1} u\right)+t^{-1}\left(c_{2} u-3 b_{1} u\right) \\
& +\left(c_{1} u+6 a_{0}\right)+(\text { higher term }) .
\end{aligned}
$$

Since $v_{o}\left(\alpha_{022}\right) \geq 1$, we obtain $c_{4}=6 a_{2}$ and $c_{3}=6 a_{1}$. By these equalities and $u=1+(\lambda+$ 1) $t^{2}-\lambda t^{4} u^{-1}$, we have

$$
\begin{aligned}
\alpha_{022}= & -6 a_{2} t^{-1}\left((\lambda+1) u-\lambda t^{2}\right)+6 a_{1}\left((\lambda+1) u-\lambda t^{2}\right)+t^{-1}\left(c_{2} u-3 b_{1} u\right) \\
& \left.+\left(c_{1} u+6 a_{0}\right)+\text { (higher term }\right) \\
= & t^{-1}\left(-6 a_{2}(\lambda+1) u+c_{2} u-3 b_{1} u\right)+\left(6 a_{1}(\lambda+1) u+c_{1} u+6 a_{0}\right)+\text { (higher term) } .
\end{aligned}
$$

Thus, we obtain the relations $-6 a_{2}(\lambda+1)+c_{2}-3 b_{1}=0$ and $6 a_{1}(\lambda+1)+c_{1}+6 a_{0}=0$. Similarly, we obtain the following relations:

$$
\begin{gathered}
b_{3}=4 a_{2}, \quad b_{2}=4 a_{1}, \quad b_{1}=-\frac{8 a_{2}(\lambda+1)}{3}, \quad b_{0}=\frac{4 a_{2} \lambda}{3}, \quad c_{4}=6 a_{2}, \quad c_{3}=6 a_{1}, \\
c_{2}=-2 a_{2}(\lambda+1), \quad c_{1}=-6 a_{0}-6 a_{1}(\lambda+1), \quad c_{0}=2\left(a_{0}(\lambda+1)+2 a_{1} \lambda\right), \\
d_{5}=4 a_{2}, \quad d_{4}=4 a_{1}, \quad d_{3}=-4 a_{2}(\lambda+1), \quad d_{2}=-4\left(2 a_{0}+a_{1}(\lambda+1)\right), \\
d_{1}=4 a_{2} \lambda, \quad d_{0}=0, \quad e_{6}=a_{2}, \quad e_{5}=a_{1}, \quad e_{4}=-\frac{2 a_{2}(\lambda+1)}{3}, \\
e_{3}=-3 a_{0}-2 a_{1}(\lambda+1), \quad e_{2}=-\frac{a_{2}(\lambda-1)^{2}}{3}, \quad e_{1}=(\lambda+1)\left(2 a_{0}+a_{1}(\lambda+1)\right), \\
e_{0}=a_{0}(\lambda-1)^{2}, \quad f_{2}=\frac{4 a_{2}(\lambda+1)}{3}, \quad g_{3}=4 a_{2}(\lambda+1), \quad g_{2}=3 f_{1},
\end{gathered}
$$




$$
\begin{gathered}
g_{1}=-4 a_{2}\left(\lambda^{2}+\lambda+1\right), \quad g_{0}=2 a_{2} \lambda(\lambda+1), \quad h_{4}=4 a_{2}(\lambda+1), \quad h_{3}=3 f_{1}, \\
h_{2}=-4 a_{2}\left(\lambda^{2}+1\right), \quad h_{1}=-3\left(f_{0}+(\lambda+1) f_{1}\right), \quad h_{0}=f_{0}(\lambda+1)+2 f_{1} \lambda, \\
i_{5}=\frac{4 a_{2}(\lambda+1)}{3}, \quad i_{4}=f_{1}, \quad i_{3}=-\frac{4 a_{2}\left(2 \lambda^{2}+\lambda+2\right)}{3}, \quad i_{2}=-2 f_{0}-f_{1}(\lambda+1), \\
i_{1}=\frac{2 a_{2}\left(2 \lambda^{3}+\lambda^{2}+\lambda+2\right)}{3}, \quad i_{0}=-\frac{2 a_{2} \lambda(\lambda-1)^{2}}{3}, \quad j_{2}=2 a_{2} \lambda, \\
j_{1}=-3 f_{0}+2 a_{1} \lambda+4 a_{0}(\lambda+1), \quad j_{0}=\left(-6 a_{0}+f_{1}\right) \lambda+2 f_{0}(\lambda+1), \quad k_{3}=4 a_{2} \lambda, \\
k_{2}=-6 f_{0}+4 a_{1} \lambda+8 a_{0}(\lambda+1), \quad k_{1}=-4 a_{2} \lambda(\lambda+1), \quad k_{0}=4 a_{2} \lambda^{2}, \quad l_{4}=2 a_{2} \lambda, \\
l_{3}=-3 f_{0}+2 a_{1} \lambda+4 a_{0}(\lambda+1), \quad l_{2}=-2 a_{2} \lambda(\lambda+1), \\
l_{1}=f_{0}(\lambda+1)-2 a_{2}\left(2 \lambda^{2}+\lambda+2\right)-\lambda\left(f_{1}+2 a_{1}(\lambda+1)\right), \\
l_{0}=2 f_{0}\left(\lambda^{2}-\lambda+1\right)+\lambda\left(f_{1}(\lambda+1)-4 a_{1} \lambda-2 a_{0}(\lambda+1)\right), \quad \\
m_{2}=0, \quad m_{1}=\left(8 a_{0}-f_{1}\right) \lambda-2 f_{0}(\lambda+1), \quad m_{0}=\lambda\left(3 f_{0}+8 a_{1} \lambda\right), \quad n_{3}=0, \\
n_{2}=\left(8 a_{0}-f_{1}\right) \lambda-2 f_{0}(\lambda+1), \quad n_{1}=-\frac{4 a_{2} \lambda^{2}}{3}, \quad n_{0}=\frac{2 a_{2} \lambda^{2}(\lambda+1)}{3}, \quad p_{2}=-\frac{a_{2} \lambda^{2}}{3}, \\
p_{1}=-\lambda\left(f_{0}+3 a_{1} \lambda\right), \quad p_{0}=\lambda^{2}\left(5 a_{0}-f_{1}+4 a_{1}(\lambda+1)\right) .
\end{gathered}
$$

By the above relations, we see that $b_{1}, b_{2}, \ldots, e_{5}, e_{6}, f_{2}, g_{0}, g_{1}, \ldots, p_{1}, p_{2}$ are the linear combinations of $a_{0}, a_{1}, a_{2}, f_{0}, f_{1}$ with coefficients in $\boldsymbol{C}$. So, $\beta_{i j k}$ are the linear combinations of $a_{0}, a_{1}, a_{2}, f_{0}, f_{1}$ with coefficients in rational functions of $E$. By replacing $\beta_{i j k}$ in $\left.\gamma\right|_{U_{Z}}$ with such linear combinations of $a_{0}, a_{1}, a_{2}, f_{0}$ and $f_{1}$, any element $\gamma \in H^{0}\left(\boldsymbol{P}_{E}(V), \mathcal{O}_{\boldsymbol{P}_{E}(V)}(4 H-\right.$ $\left.F_{o}\right)$ ) is represented as

$$
\begin{aligned}
\gamma & =\sum_{\substack{i, j, k \geq 0 \\
i+j+k=4}} \beta_{i j k} Z_{0}^{i} Z_{1}^{j} Z_{2}^{k} \\
& =a_{0} F_{1}+a_{1} F_{2}+f_{0} F_{3}+f_{1} F_{4}+\frac{a_{2}}{3} F_{5} .
\end{aligned}
$$

Hence, the vector space $H^{0}\left(\boldsymbol{P}_{E}(V), \mathcal{O}_{\boldsymbol{P}_{E}(V)}\left(4 H-F_{o}\right)\right)$ is generated by $F_{1}, \ldots, F_{5}$. By [3, Theorem 1.17], we see that the dimension of $H^{0}\left(\boldsymbol{P}_{E}(V), \mathcal{O}_{\boldsymbol{P}_{E(V)}}\left(4 H-F_{o}\right)\right)$ is five. Thus, the vector space $H^{0}\left(\boldsymbol{P}_{E}(V), \mathcal{O}_{\boldsymbol{P}_{E}(V)}\left(4 H-F_{o}\right)\right)$ has a basis $\left\{F_{1}, F_{2}, F_{3}, F_{4}, F_{5}\right\}$.

REMARK 4.3. Let $S$ be a Catanese-Ciliberto surface defined by $F=\sum_{i=1}^{5} s_{i} F_{i}$. Let $F(o)$ be the defining polynomial of the fiber of $S$ at $o$. Then it is easy to see that $F(o)=$ $\left.t^{-1} F\left(Y_{0},-t^{-1} Y_{0}+Y_{1}, t^{-2} Y_{0}-t^{-1} Y_{1}+t Y_{2}\right)\right|_{t=0}$. By a calculation, we obtain

$$
\begin{aligned}
F(o)= & s_{1}\left(8 \lambda^{2} Y_{0}^{3} Y_{1}+4 \lambda(\lambda+1) Y_{0}^{3} Y_{2}+12 \lambda Y_{0} Y_{1}^{2} Y_{2}+8(\lambda+1) Y_{0} Y_{1} Y_{2}^{2}+4 Y_{0} Y_{2}^{3}\right) \\
& +s_{2}\left(4 \lambda^{2}(\lambda+1) Y_{0}^{3} Y_{1}+8 \lambda^{2} Y_{0}^{3} Y_{2}-4 \lambda^{2} Y_{0} Y_{1}^{3}+4 \lambda Y_{0} Y_{1} Y_{2}^{2}\right) \\
& +s_{3}\left(-\lambda(\lambda+1) Y_{0}^{3} Y_{1}-2\left(\lambda^{2}-\lambda+1\right) Y_{0}^{3} Y_{2}-\lambda Y_{0} Y_{1}^{3}-2(\lambda+1) Y_{0} Y_{1}^{2} Y_{2}-3 Y_{0} Y_{1} Y_{2}^{2}\right) \\
& +s_{4}\left(-2 \lambda^{2} Y_{0}^{3} Y_{1}-\lambda(\lambda+1) Y_{0}^{3} Y_{2}-\lambda Y_{0} Y_{1}^{2} Y_{2}+Y_{0} Y_{2}^{3}\right) \\
& +s_{5}\left(\lambda^{2}(\lambda-1)^{2} Y_{0}^{4}-\lambda^{2} Y_{1}^{4}+6 \lambda Y_{1}^{2} Y_{2}^{2}+4(\lambda+1) Y_{1} Y_{2}^{3}+3 Y_{2}^{4}\right) .
\end{aligned}
$$


4.3. Defining equations of the Catanese-Ciliberto surfaces with unique singular fiber II. In Section 2.2, we obtained the defining equations of the unramified triple coverings of the Catanese-Ciliberto surfaces. These were given as $G$-invariant global sections of $\mathcal{O}_{\boldsymbol{P}_{\tilde{E}}}(4 \tilde{H}-$ $\left.\tilde{p}^{*}[o]\right)$. The defining equations of Catanese-Ciliberto surfaces are elements of $H^{0}\left(\boldsymbol{P}_{E}(V)\right.$, $\left.\mathcal{O}_{\boldsymbol{P}_{E}(V)}\left(4 H-p^{*} o\right)\right)$. Hence, by Lemma 4.2 , it is described as $F=\sum_{i=1}^{5} s_{i} F_{i}$. First, let us mention a result which we need later.

LEMMA 4.4. Let $\pi: V \rightarrow E$ be an indecomposable bundle of rank three and degree one. Then the group Aut $(V)$ of automorphisms $\Phi^{*}$ of $V$ satisfying $\Phi^{*} \circ \pi=\pi$ is isomorphic to $\boldsymbol{C}^{*}$.

Proof. Let $Y_{i}$ and $Z_{i}$ be as in the previous section. Let $\Phi^{*}$ be an automorphism of $V$ such that $\Phi^{*} \circ \pi=\pi$. Since $Z_{0}\left(=Y_{0}\right)$ is a unique global section of $V$ up to multiplications of complex numbers, we have $\Phi^{*} Z_{0}=c Z_{0}$ and $\Phi^{*} Y_{0}=c Y_{0}$, where $c \in C^{*}$. Since $\Phi^{*} Z_{i}$ (resp. $\left.\Phi^{*} Y_{j}\right)$ is contained in $V\left(U_{Z}\right)$ (resp. $V\left(U_{Y}\right)$ ), we can write

$$
\begin{aligned}
\Phi^{*} Y_{1} & =\alpha_{1,0} Y_{0}+\alpha_{1,1} Y_{1}+\alpha_{1,2} Y_{2}, \\
\Phi^{*} Y_{2} & =\alpha_{2,0} Y_{0}+\alpha_{2,1} Y_{1}+\alpha_{2,2} Y_{2}, \\
\Phi^{*} Z_{1} & =\beta_{1,0} Z_{0}+\beta_{1,1} Z_{1}+\beta_{1,2} Z_{2}, \\
\Phi^{*} Z_{2} & =\beta_{2,0} Z_{0}+\beta_{2,1} Z_{1}+\beta_{2,2} Z_{2},
\end{aligned}
$$

where $\alpha_{i, j}$ and $\beta_{i, j}$ are in $\mathcal{O}_{E}\left(U_{Y}\right)$ and $\mathcal{O}_{E}\left(U_{Z}\right)$, respectively. Since $Y_{1}=t^{-1} Z_{0}+Z_{1}$ and $Y_{2}=t^{-2} Z_{1}+t^{-1} Z_{2}$, by the transition relation (89), we obtain

$$
\begin{aligned}
\Phi^{*} Y_{i} & =\alpha_{i, 0} Y_{0}+\alpha_{i, 1} Y_{1}+\alpha_{i, 2} Y_{2} \\
& =\left(\alpha_{i, 0}+t^{-1} \alpha_{i, 1}\right) Z_{0}+\left(\alpha_{i, 1}+t^{-2} \alpha_{i, 2}\right) Z_{1}+t^{-1} \alpha_{i, 2} Z_{2} .
\end{aligned}
$$

Also, by (89), we have

$$
\begin{aligned}
\Phi^{*} Y_{1} & =t^{-1} \Phi^{*} Z_{0}+\Phi^{*} Z_{1} \\
& =\left(c t^{-1}+\beta_{1,0}\right) Z_{0}+\beta_{1,1} Z_{1}+\beta_{1,2} Z_{2}, \\
\Phi^{*} Y_{2} & =t^{-2} \Phi^{*} Z_{1}+t^{-1} \Phi^{*} Z_{2} \\
& =\sum_{j=0}^{2}\left(t^{-2} \beta_{1, j}+t^{-1} \beta_{2, j}\right) Z_{j} .
\end{aligned}
$$

Hence, by comparing coefficients of $Z_{0}, Z_{1}$ and $Z_{2}$ for $\Phi^{*} Y_{1}$ and $\Phi^{*} Y_{2}$, we obtain the following relations.

$$
\begin{gathered}
\alpha_{1,0}+t^{-1} \alpha_{1,1}=c t^{-1}+\beta_{1,0}, \\
\alpha_{1,1}+t^{-2} \alpha_{1,2}=\beta_{1,1}, \\
t^{-1} \alpha_{1,2}=\beta_{1,2}, \\
\alpha_{2,0}+t^{-1} \alpha_{2,1}=t^{-2} \beta_{1,0}+t^{-1} \beta_{2,0}, \\
\alpha_{2,1}+t^{-2} \alpha_{2,2}=t^{-2} \beta_{1,1}+t^{-1} \beta_{2,1},
\end{gathered}
$$




$$
t^{-1} \alpha_{2,2}=t^{-2} \beta_{1,2}+t^{-1} \beta_{2,2} .
$$

Since $\alpha_{1,2}$ is regular at $o \in U_{Y}$, we see that $\beta_{1,2} \in H^{0}\left(E, \mathcal{O}_{E}(o)\right)=C$ by the equality (107). If $\beta_{1,2} \neq 0$, then $v_{o}\left(\alpha_{1,1}\right)=v_{o}\left(\beta_{1,1}-t^{-1} \beta_{1,2}\right)=-1$. So we obtain $\beta_{1,2}=\alpha_{1,2}=0$. By the equality $\alpha_{1,1}=\beta_{1,1}$, we have $\beta_{1,1} \in H^{0}\left(E, \mathcal{O}_{E}(o)\right)=C$. Similarly, by equalities (105)-(110), we obtain the following relations:

$$
\begin{aligned}
& \alpha_{1,2}=\beta_{1,2}= \alpha_{1,0}=\beta_{1,0}=\alpha_{2,1}=\beta_{2,1}=\alpha_{2,0}=\beta_{2,0}=0, \\
& \alpha_{1,1}=\beta_{1,1}=\alpha_{2,2}=\beta_{2,2}=c,
\end{aligned}
$$

i.e., we have $\Phi^{*} Z_{1}=c Z_{1}$ and $\Phi^{*} Z_{2}=c Z_{2}$, i.e., $\Phi^{*}$ is the multiplication of the constant c.

Now we give the defining equations of these surfaces in $\boldsymbol{P}_{E}$.

Lemma 4.5. Let $\lambda$ be a complex number in $\boldsymbol{C} \backslash\{0,1\}$ and $E \subset \boldsymbol{P}^{2}$ the elliptic curve defined by the equation $Y^{2} Z=X(X-Z)(X-\lambda Z)$ and $D_{\lambda}(T)$ the quartic polynomial $\lambda^{2} T^{4}-6 \lambda T^{2}-4(\lambda+1) T-3$ with the variable $T$. Let $\xi$ be a complex number satisfying the equality $D_{\lambda}(\xi)=\lambda^{2} \xi^{4}-6 \lambda \xi^{2}-4(\lambda+1) \xi-3=0$. Let $L_{\lambda}(\xi)$ be the matrix defined as follows:

$$
\begin{aligned}
& L_{\lambda}(\xi)= \\
& \left(\begin{array}{cccc}
-8 \lambda^{2} \xi-4 \lambda(\lambda+1) & -4 \lambda^{2}(\lambda+1) \xi-8 \lambda^{2} & \lambda(\lambda+1) \xi+2\left(\lambda^{2}-\lambda+1\right) & 2 \lambda^{2} \xi+\lambda(\lambda+1) \\
-12 \lambda & 12 \lambda^{2} \xi & 3 \lambda \xi+2(\lambda+1) & \lambda \\
-12 \lambda \xi-8(\lambda+1) & -4 \lambda & 2(\lambda+1) \xi+3 & \lambda \xi \\
8(\lambda+1) \xi+12 & 4 \lambda \xi & -3 \xi & 3
\end{array}\right) .
\end{aligned}
$$

Let $\left(s_{1}, s_{2}, s_{3}, s_{4}\right)$ be a non-zero vector with $L_{\lambda}(\xi)^{\mathrm{t}}\left(s_{1}, s_{2}, s_{3}, s_{4}\right)=0$. Then we obtain the following.

(i) For every $\lambda \in C \backslash\{0,1\}$, the quartic equation $D_{\lambda}(T)=0$ has four distinct solutions. The rank of $L_{\lambda}(\xi)$ is three for any $\lambda \in C \backslash\{0,1\}$ and $\xi$ satisfying the equality $D_{\lambda}(\xi)=0$. In particular, There exist four choices of $\left(s_{1}, s_{2}, s_{3}, s_{4}\right)$ up to multiplications of complex numbers for each $\lambda \in \boldsymbol{C} \backslash\{0,1\}$.

(ii) The equations $\sum_{i=1}^{4} s_{i} F_{i}=0$ define Catanese-Ciliberto surfaces $S$ of type I with $\operatorname{Alb}(S) \cong E$. Conversely, a Catanese-Ciliberto surface of type I with $\operatorname{Alb}(S) \cong E$ is defined by one of four equations $\sum_{i=1}^{4} s_{i} F_{i}=0$.

Proof. Let $S$ be a Catanese-Ciliberto surface of type $\mathrm{I}$ with $\operatorname{Alb}(S) \cong E$. We use the notation such as $\iota, \bar{\imath}$ and $\boldsymbol{P}_{\tilde{E}}$ in Section 2. By Proposition 2.8 and Remark 2.7, the unramified triple covering $\tilde{S}$ of $S$ has an automorphism obtained by restricting an automorphism $\bar{\imath}$ of $\boldsymbol{P}_{\tilde{E}}$ which commutes with the involution $\iota$ of the elliptic curve $\tilde{E}$. Moreover, the defining equation of $\tilde{S}$ in $\boldsymbol{P}_{\tilde{E}}$ is $\tilde{\iota}^{*}$-invariant. Since $G=\left\{o, o^{\prime}, o^{\prime \prime}\right\}$ acts on $\boldsymbol{P}_{\tilde{E}}$ as translations and we have $\iota \circ T_{o^{\prime}}=T_{o^{\prime \prime}} \circ \iota$ and $\tilde{S} / G=S, S$ has an automorphism obtained by restricting an automorphism of $\boldsymbol{P}_{E}$ which commutes with the involution of the elliptic curve $E$. 
Let $\iota_{2}$ be the involution of $E$ and $\overline{\iota_{2}}$ an automorphism of $\boldsymbol{P}_{E}$ defined by $\bar{\iota}_{2} * Z_{0}=$ $Z_{0}, \bar{\iota}_{2}^{*} Z_{1}=-Z_{1}$ and $\bar{\iota}_{2}^{*} Z_{2}=Z_{2}$. Then, by the properties $\iota_{2}^{*} x=x, \iota_{2}^{*} y=-y$, it is clear that $\overline{\iota_{2}}$ commutes with $\iota_{2}$. By Lemma 4.4, an automorphism of $P_{E}$ which commutes with $\iota_{2}$ is equal to $\overline{\iota_{2}}$.

Let $F=\sum_{i=1}^{5} s_{i} F_{i}$ be the defining equation of $S$. Since $\bar{\iota}_{2}^{*} Z_{0}=Z_{0}, \bar{\iota}_{2}{ }^{*} Z_{1}=-Z_{1}$ and $\bar{\iota}_{2}^{*} Z_{2}=Z_{2}$, we have $\bar{\iota}_{2}^{*} F_{5}=-F_{5}$ and $\bar{\iota}_{2}^{*} F_{i}=F_{i}$ for $i=1,2,3,4$. $s_{5}=0$.

Since $S$ is $\overline{\iota_{2}}$-invariant, we have $c F=\bar{\iota}_{2}^{*} F=\sum_{i=1}^{4} s_{i} F_{i}-s_{5} F_{5}$ for $c \in C^{*}$, i.e., we get

By Proposition 2.8, the unique singular fiber of $S$ is at the point $o$. By Remark 4.3, the defining equation of the fiber of $S$ at $o$ is written as follows:

$$
\begin{aligned}
F(o)= & s_{1}\left(8 \lambda^{2} Y_{0}^{3} Y_{1}+4 \lambda(\lambda+1) Y_{0}^{3} Y_{2}+12 \lambda Y_{0} Y_{1}^{2} Y_{2}+8(\lambda+1) Y_{0} Y_{1} Y_{2}^{2}+4 Y_{0} Y_{2}^{3}\right) \\
& +s_{2}\left(4 \lambda^{2}(\lambda+1) Y_{0}^{3} Y_{1}+8 \lambda^{2} Y_{0}^{3} Y_{2}-4 \lambda^{2} Y_{0} Y_{1}^{3}+4 \lambda Y_{0} Y_{1} Y_{2}^{2}\right) \\
& +s_{3}\left(-\lambda(\lambda+1) Y_{0}^{3} Y_{1}-2\left(\lambda^{2}-\lambda+1\right) Y_{0}^{3} Y_{2}-\lambda Y_{0} Y_{1}^{3}-2(\lambda+1) Y_{0} Y_{1}^{2} Y_{2}-3 Y_{0} Y_{1} Y_{2}^{2}\right) \\
& +s_{4}\left(-2 \lambda^{2} Y_{0}^{3} Y_{1}-\lambda(\lambda+1) Y_{0}^{3} Y_{2}-\lambda Y_{0} Y_{1}^{2} Y_{2}+Y_{0} Y_{2}^{3}\right) .
\end{aligned}
$$

Moreover, this fiber is a quartic curve which consists of four lines intersecting at a point. One of these four lines is defined by $Y_{0}=0$. Set $\bar{F}=F(o) / Y_{0}$. Then the equation $\bar{F}=0$ defines a cubic curve with a triple point on the line $Y_{0}=0$. Thus, there exists a point satisfying the following linear relations in $s_{1}, s_{2}, s_{3}, s_{4}$ :

$$
\begin{aligned}
\left.\partial_{Y_{0}} \partial_{Y_{0}} \bar{F}\right|_{Y_{0}=0}= & -2\left(\left(-8 \lambda^{2} Y_{1}-4 \lambda(\lambda+1) Y_{2}^{2}\right) s_{1}+\left(-4 \lambda^{2}(\lambda+1) Y_{1}-8 \lambda^{2} Y_{2}\right) s_{2}\right. \\
& \left.+\left(\lambda(\lambda+1) Y_{1}+2\left(\lambda^{2}-\lambda+1\right) Y_{2}\right) s_{3}+\left(2 \lambda^{2} Y_{1}+(\lambda+1) Y_{2}\right) s_{4}\right)=0, \\
\left.\partial_{Y_{1}} \partial_{Y_{1}} \bar{F}\right|_{Y_{0}=0}= & 2\left(12 \lambda Y_{2} s_{1}-12 \lambda^{2} Y_{1} s_{2}-\left(3 \lambda Y_{1}+2(\lambda+1) Y_{2}\right) s_{3}-\lambda Y_{2} s_{4}\right)=0, \\
\left.\partial_{Y_{1}} \partial_{Y_{2}} \bar{F}\right|_{Y_{0}=0}= & 2\left(\left(12 \lambda Y_{1}+8(\lambda+1) Y_{2}\right) s_{1}+4 \lambda Y_{2} s_{2}-\left(2(\lambda+1) Y_{1}+3 Y_{2}\right) s_{3}-\lambda Y_{1} s_{4}\right)=0, \\
\left.\partial_{Y_{2}} \partial_{Y_{2}} \bar{F}\right|_{Y_{0}=0}= & 2\left(\left(8(\lambda+1) Y_{1}+12 Y_{2}\right) s_{1}+4 \lambda Y_{1} s_{2}-3 Y_{1} s_{3}+3 Y_{2} s_{4}\right)=0 .
\end{aligned}
$$

By using the matrix $L_{\lambda}$ of the coefficients, we write these equalities as

$$
\left.{ }^{\mathrm{t}}\left(\partial_{Y_{0}} \partial_{Y_{0}} \bar{F}, \partial_{Y_{1}} \partial_{Y_{1}} \bar{F}, \partial_{Y_{1}} \partial_{Y_{2}} \bar{F}, \partial_{Y_{2}} \partial_{Y_{2}} \bar{F}\right)\right|_{Y_{0}=0}=L_{\lambda}{ }^{\mathrm{t}}\left(s_{1}, s_{2}, s_{3}, s_{4}\right)=0 .
$$

The determinant of the matrix $L_{\lambda}$ is calculated to be

$$
\begin{gathered}
-192(\lambda-1)^{2} \lambda^{2}\left(\lambda^{2} Y_{1}^{4}-6 \lambda Y_{1}^{2} Y_{2}^{2}-4(\lambda+1) Y_{1} Y_{2}^{3}-3 Y_{2}^{4}\right) \\
=-192(\lambda-1)^{2} \lambda^{2} D_{\lambda}\left(Y_{1} / Y_{2}\right) Y_{2}^{4} .
\end{gathered}
$$

We obtain the matrix $L_{\lambda}(\xi)$ by replacing $Y_{1}$ and $Y_{2}$ by $\xi$ and 1 . Then $\left(s_{1}, s_{2}, s_{3}, s_{4}\right)$ satisfies $L_{\lambda}(\xi)^{\mathrm{t}}\left(s_{1}, s_{2}, s_{3}, s_{4}\right)=0$ if $(0: \xi: 1)$ is the triple point of the cubic curve $\bar{F}=0$. Hence, the defining equation $F=\sum_{i=1}^{4} s_{i} F_{i}$ of $S$ satisfies $L_{\lambda}(\xi)^{\mathrm{t}}\left(s_{1}, s_{2}, s_{3}, s_{4}\right)=0$, where $\xi$ is a solution of the equation $D_{\lambda}(T)=0$.

Furthermore, the equation $D_{\lambda}(T)=0$ in $T$ does not have multiple solutions. Thus, the number of solutions of $D_{\lambda}(T)=0$ is four. By an easy calculation, we see that the rank of $L_{\lambda}(\xi)$ is three. So a vector $\left(s_{1}, s_{2}, s_{3}, s_{4}\right)$ is determined uniquely up to multiplications of complex numbers for $\lambda \in \boldsymbol{C} \backslash\{0,1\}$ and $\xi \in \boldsymbol{C}$ satisfying the equality $D_{\lambda}(\xi)=0$. 
Conversely, we assume that $\xi$ and $\left(s_{1}, s_{2}, s_{3}, s_{4}\right)$ satisfy equalities $D_{\lambda}(\xi)=0$ and $L_{\lambda}(\xi)^{\mathrm{t}}\left(s_{1}, s_{2}, s_{3}, s_{4}\right)=0$. Then the surface $S$ defined by $\sum_{i=1}^{4} s_{i} F_{i}=0$ has a fiber with a quadruple point. Therefore, we have only to show that $S$ is non-singular. Since the unramified triple covering of $S$ has three singular fibers with a quadruple point, this is isomorphic to $\tilde{S}_{1}$ or $\tilde{S}_{\zeta}$. Note that $\tilde{S}_{1}$ and $\tilde{S}_{\zeta}$ are as in Section 2. By Example 2.2 and Lemma 3.4, $\tilde{S}_{1}$ and $\tilde{S}_{\zeta}$ are non-singular. Hence, $S$ is non-singular, i.e., $S$ is the Catanese-Ciliberto surface of type I. Therefore, we complete the proof.

4.4. Completion of the proof of Theorem 0.2. Let $s(\xi)=\left(s(\xi)_{1}, s(\xi)_{2}, s(\xi)_{3}, s(\xi)_{4}\right)$ be a non-zero vector satisfying $L_{\lambda}(\xi)^{\mathrm{t}} s(\xi)=0$ for each solution $\xi$ of the equation $D_{\lambda}(\xi)=$ $\lambda^{2} \xi^{4}-6 \lambda \xi^{2}-4(\lambda+1) \xi-3=0$. Set $F_{\xi}=\sum_{i=1}^{4} s(\xi)_{i} F_{i}$. We know that the surface $S_{\xi}$ defined by $F_{\xi}=0$ in $\boldsymbol{P}_{E}(V)$ is a Catanese-Ciliberto surface of type I. Hence, it suffices to consider the isomorphic classes among the four surfaces $S_{\xi}$. Set $\mathrm{CCI}_{\lambda}=\left\{S_{\xi} \mid \xi \in \boldsymbol{C}, D_{\lambda}(\xi)=0\right\}$.

LEMMA 4.6. Let $\xi_{1}$ and $\xi_{2}$ be solutions of $D_{\lambda}(T)=0$. If there exists an isomorphism $\Phi: S_{\xi_{1}} \rightarrow S_{\xi_{2}}$, then $\Phi$ induces an automorphism $\varphi$ of $E$ with $\varphi(o)=o$, and $\Phi$ is the restriction of an automorphism of $\boldsymbol{P}_{E}$ which commutes with $\varphi$.

Proof. Since $\operatorname{Alb}\left(S_{\xi_{1}}\right)=\operatorname{Alb}\left(S_{\xi_{2}}\right)=E, \Phi$ induces an automorphism $\varphi: E \rightarrow E$ by the universality of the Albanese maps. Since each of $S_{\xi_{1}}$ and $S_{\xi_{2}}$ has a unique singular fiber at $o$, we have $\varphi(o)=o$.

Let $i_{\xi_{j}}: S_{\xi_{j}} \hookrightarrow \boldsymbol{P}_{E}$ be the natural closed immersion for $j=1,2$. By the adjunction formula, we have $i_{\xi_{j}}{ }^{*} \mathcal{O}_{\boldsymbol{P}_{E}}(H) \cong K_{S_{\xi_{j}}}$ since $S_{\xi_{j}}$ and $K_{\boldsymbol{P}_{E}}$ are linearly equivalent to $4 H-F_{O}$ and $-3 H+F_{o}$, respectively. In other words, $i_{\xi_{j}}$ is the relative canonical map of $S_{\xi_{j}}$. Since $\Phi$ is an isomorphism from $S_{\xi_{1}}$ to $S_{\xi_{2}}$, we have an isomorphism $\Phi^{*} K_{S_{\xi_{2}}} \stackrel{\sim}{\rightarrow} K_{S_{\xi_{1}}}$. Hence, we have an isomorphism $\varphi^{*}\left(\left.p\right|_{S_{\xi_{2}}}\right)_{*} K_{S_{\xi_{2}}} \stackrel{\sim}{\rightarrow}\left(\left.p\right|_{S_{\xi_{1}}}\right)_{*} \Phi^{*} K_{S_{\xi_{2}}} \stackrel{\sim}{\rightarrow}\left(\left.p\right|_{S_{\xi_{1}}}\right)_{*} K_{S_{\xi_{1}}}$ which we denote by $\bar{\Phi}^{*}$. By [4, II, Proposition 7.12], $\bar{\Phi}^{*}$ induces the automorphism $\bar{\Phi}$ of $\boldsymbol{P}_{E}$ satisfying the following commutative diagram:

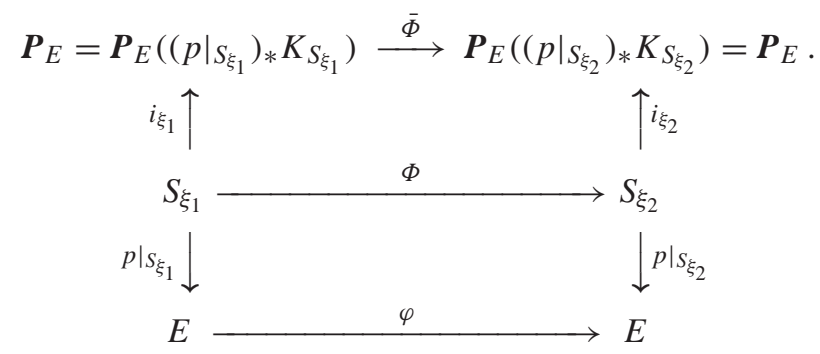

Note that $\left(\left.p\right|_{\xi_{j}}\right)_{*} K_{S_{\xi_{j}}}$ coincide with an indecomposable bundle $V$ of rank three and degree one with $\operatorname{det} V \cong \mathcal{O}_{E}(o)$ by the construction of $S_{\xi_{j}}$.

By the above lemma, we have the following consequence.

LEMMA 4.7. If $E$ has no automorphism of complex multiplication type, then there exist no isomorphisms among the four surfaces in $\mathrm{CCI}_{\lambda}$. 
ProOF. Let $\xi_{1}$ and $\xi_{2}$ be solutions of $D_{\lambda}(T)=0$. It suffices to show $\xi_{1}=\xi_{2}$ if there exists an isomorphism $\Phi: S_{\xi_{1}} \rightarrow S_{\xi_{2}}$. It is easy to see that $s\left(\xi_{1}\right)_{1}, s\left(\xi_{2}\right)_{1} \neq 0$. We can assume that $s\left(\xi_{1}\right)_{1}=s\left(\xi_{2}\right)_{1}=1$. By Lemma 4.6, $\Phi$ is the restriction of an automorphism $\bar{\Phi}$ of $\boldsymbol{P}_{E}$ which commutes with an automorphism $\varphi$ of $E$ leaving $o$ fixed. Since $\bar{\Phi}$ gives an isomorphism from $S_{\xi_{1}}$ to $S_{\xi_{2}}$, we have $F_{\xi_{1}}=c \bar{\Phi}^{*} F_{\xi_{2}}\left(c \in C^{*}\right)$, where we also denote by $\bar{\Phi}^{*}$ the automorphism of $\operatorname{Sym}^{4} V$ induced by $\bar{\Phi}^{*}$.

Since $E$ has no automorphism of complex multiplication type, $\varphi$ is equal to $\operatorname{id}_{E}$ or $\iota_{2}$, where $\iota_{2}$ is the involution of $E$.

If $\varphi=\mathrm{id}_{E}$, by Lemma 4.4 , we see that $\bar{\Phi}^{*} Z_{0}=d Z_{0}, \bar{\Phi}^{*} Z_{1}=d Z_{1}$ and $\bar{\Phi}^{*} Z_{2}=d Z_{2}$ for $d \in C^{*}$. Therefore, we have $\bar{\Phi}^{*} F_{i}=d^{4} F_{i}$ for $i=1,2,3,4,5$. We obtain $F_{\xi_{1}}=$ $c \bar{\Phi}^{*} F_{\xi_{2}}=c d^{4} F_{\xi_{2}}$. By the assumption $s\left(\xi_{1}\right)_{1}=s\left(\xi_{2}\right)_{1}=1$, we have $c d^{4}=1$ and $s\left(\xi_{1}\right)_{i}=$ $s\left(\xi_{2}\right)_{i}$ for $i=1,2,3,4$. Thus, we have $\xi_{1}=\xi_{2}$ if $\varphi=\operatorname{id}_{E}$.

We consider the case where $\varphi=\iota_{2}$. Let $\bar{l}_{2}$ be an automorphism of $\boldsymbol{P}_{E}$ as in the proof of Lemma 4.5. By Lemma 4.4, we see that $\bar{\Phi}=\overline{\iota_{2}}$, i.e., $\bar{\Phi}^{*} Z_{0}=d Z_{0}, \bar{\Phi}^{*} Z_{1}=-d Z_{1}$ and $\bar{\Phi}^{*} Z_{2}=d Z_{2}$ for $d \in C^{*}$. Therefore, we have $\bar{\Phi}^{*} F_{5}=-d^{4} F_{5}$ and $\bar{\Phi}^{*} F_{i}=d^{4} F_{i}$ for $i=1,2,3,4$. We obtain $F_{\xi_{1}}=c \bar{\Phi}^{*} F_{\xi_{2}}=c d^{4} F_{\xi_{2}}$.

Now, we consider the case where the Albanese torus $E$ has an automorphism of complex multiplication type. Since $E$ is defined by the equation $Y^{2} Z=X(X-Z)(X-\lambda Z)$, we have to consider the cases where $\lambda=-1$ and $\lambda=(1+\sqrt{-3}) / 2$. First we consider the case $\lambda=-1$.

LEMMA 4.8. Assume that $\lambda=-1$. Then, there exist four solutions $\pm \xi_{0}, \pm \xi_{1}$ of the equality $D_{-1}(T)=0$, and there exist isomorphisms $S_{\xi_{0}} \cong S_{-\xi_{0}}$ and $S_{\xi_{1}} \cong S_{-\xi_{1}}$, while $S_{\xi_{0}}$ and $S_{\xi_{1}}$ are not isomorphic.

Proof. First, we describe $\left(s(\xi)_{1}: s(\xi)_{2}: s(\xi)_{3}: s(\xi)_{4}\right)$ for a solution $\xi$ of the equation $D_{-1}(T)=0$. By substituting -1 for $\lambda$ of $L_{\lambda}(\xi)$ in Lemma 4.5 , we get

$$
L_{-1}(\xi)=\left(\begin{array}{cccc}
-8 \xi & -8 & 6 & 2 \xi \\
12 & 12 \xi & -3 \xi & -1 \\
12 \xi & 4 & 3 & -\xi \\
12 & -4 \xi & -3 \xi & 3
\end{array}\right) .
$$

From this matrix, we have

$$
\begin{aligned}
& \left(s(\xi)_{1}: s(\xi)_{2}: s(\xi)_{3}: s(\xi)_{4}\right)=\left(3\left(\xi^{2}-1\right): 6 \xi:-4 \xi\left(\xi^{2}-1\right): 24 \xi^{2}\right), \\
\text { i.e., } F_{\xi}= & 3\left(\xi^{2}-1\right) F_{1}+6 \xi F_{2}-4 \xi\left(\xi^{2}-1\right) F_{4}+24 \xi^{2} F_{5} .
\end{aligned}
$$

Let $\left\{\xi_{0},-\xi_{0}, \xi_{1},-\xi_{1}\right\}$ be the set of solutions of $D_{-1}(T)=T^{4}+6 T^{2}-3=0$. We use the notation as in Lemma 4.6. By Lemma 4.6, if $S_{\xi_{0}}$ is isomorphic to another surface $S_{\xi}$, then there exists a unique automorphism $\bar{\Phi}$ of $\boldsymbol{P}_{E}$ which commutes with an automorphism $\varphi$ of $E$ leaving $o$ fixed such that $\bar{\Phi}^{*} F\left(\xi_{0}\right)=c F(\xi)$ for $c \in C^{*}$.

Let $\iota_{4}: E \rightarrow E$ be the automorphism of $E$ with $\iota_{4}^{*}(X / Z)=-X / Z$ and $\iota_{4}^{*}(Y / Z)=$ $\sqrt{-1} Y / Z$. Then we see that the order of $\iota_{4}$ is four and the group of automorphisms of $E$ is equal to the set $\left\{\operatorname{id}_{E}, \iota_{2}, \iota_{4}, \iota_{4} \circ \iota_{2}\right\}$. 
In the cases where $\varphi=\operatorname{id}_{E}$ and $\varphi=\iota_{2}$, we see that $\bar{\Phi}^{*} F\left(\xi_{0}\right)=c F\left(\xi_{0}\right)$ for $c \in C^{*}$ similarly as in the proof of Lemma 4.7.

We consider the case where $\varphi=\iota_{4}$. Let $\overline{l_{4}}$ be the automorphism of $\boldsymbol{P}_{E}$ defined by $\bar{\iota}_{4}^{*} Z_{0}=Z_{0}, \bar{\iota}_{4}^{*} Z_{1}=-\sqrt{-1} Z_{1}$ and $\bar{\iota}_{4}^{*} Z_{2}=-Z_{2}$. Then, by the properties $\iota_{4}^{*} x=-x, \iota_{4}^{*} y=$ $\sqrt{-1} y$, it is clear that $\iota_{4}$ commutes with $\iota_{4}$. By Lemma 4.4, an automorphism of $\boldsymbol{P}_{E}$ which commutes with $\iota_{4}$ is equal to $\overline{\iota_{4}}$, i.e., $\bar{\Phi}=\overline{\iota_{4}}$. We obtain the following equalities:

$$
\begin{gathered}
\bar{\iota}^{*} F_{1}=F_{1}, \quad \bar{\iota}^{*} F_{2}=-F_{2}, \quad \bar{\iota}^{*} F_{3}=-F_{3}, \\
\bar{\iota}^{*} F_{4}=F_{4}, \quad \bar{\iota}^{*} F_{5}=\sqrt{-1} F_{5} .
\end{gathered}
$$

Therefore, we have

$$
\bar{\iota}_{4}^{*} F_{\xi_{0}}=\left\{3\left(\left(-\xi_{0}\right)^{2}-1\right) F_{1}+6\left(-\xi_{0}\right) F_{2}-4\left(-\xi_{0}\right)\left(\left(-\xi_{0}\right)^{2}-1\right) F_{4}+24\left(-\xi_{0}\right)^{2} F_{5}\right\},
$$

i.e., $\bar{\iota}_{4}^{*} F_{\xi_{0}}=F_{-\xi_{0}}$. Hence, $S_{\xi_{0}}$ and $S_{-\xi_{0}}$ are isomorphic to each other.

In the case where $\varphi=\iota_{2} \circ \iota_{4}$, we see that $\bar{\Phi}=\overline{\iota_{2}} \circ \overline{\iota_{4}}$. Since $\bar{\iota}_{2}^{*} F_{\xi_{0}}=F_{\xi_{0}}$ and $\bar{\iota}^{*} F_{\xi_{0}}=F_{-\xi_{0}}$, we obtain $\bar{\Phi}^{*} F_{\xi_{0}}=F_{-\xi_{0}}$.

By the above argument, for any automorphism $\varphi$ of $E$ leaving $o$ fixed, we have $\bar{\Phi}^{*} F\left(\xi_{0}\right)$ $=F\left(\xi_{0}\right)$ or $F\left(-\xi_{0}\right)$. Thus, $S_{\xi_{0}}$ is not isomorphic to $S_{\xi_{1}}$ and $S_{-\xi_{1}}$.

Next, we consider the case $\lambda=(1+\sqrt{-3}) / 2$.

Lemma 4.9. Assume that $\lambda=(1+\sqrt{-3}) / 2$. Then $\xi_{0}=\lambda-2$ is a solution of the equation $D_{\lambda}(T)=0$, and $S_{\xi_{0}}$ is not isomorphic to the other elements of $\mathrm{CCI}_{\lambda}$. Let $\xi_{1}, \xi_{2}, \xi_{3}$ be the other solutions. Then $S_{\xi_{1}}, S_{\xi_{2}}$ and $S_{\xi_{3}}$ are mutually isomorphic.

Proof. We use the notation as in Lemma 4.6. Let $\iota_{6}$ be the automorphism of $E$ with $\iota_{6}^{*}(X / Z)=-\lambda(X / Z-1), \iota_{6}^{*}(Y / Z)=-Y / Z$. Let $\overline{l_{6}}$ be the automorphism of $\boldsymbol{P}_{E}$ defined by $\overline{l \sigma}^{*} Z_{0}=Z_{0}, \bar{\iota}_{6}^{*} Z_{1}=-\lambda^{2} Z_{1}$ and $\overline{l \sigma}^{*} Z_{2}=-\lambda\left(Z_{0}+Z_{2}\right)$. Then, by the properties $\iota_{6}^{*} x=-\lambda(x-1), \iota_{6}^{*} y=-y$, it is clear that $\overline{l_{6}}$ commutes with $\iota_{6}$. By Lemma 4.4, an automorphism of $P_{E}$ which commutes with $\iota_{6}$ is equal to $\overline{l_{6}}$, i.e., $\bar{\Phi}=\overline{l_{6}}$. By these properties, we obtain the following equalities:

$$
\begin{gathered}
\bar{\iota}^{*} F_{1}=\left(-\lambda F_{1}-4 \lambda F_{3}\right), \quad \bar{\iota}_{6}^{*} F_{2}=-\lambda^{2}\left(F_{1}-F_{2}+4 F_{3}-4 F_{4}\right), \\
\bar{\iota}^{*} F_{3}=F_{3}, \quad \bar{\iota}^{*} F_{4}=\lambda\left(F_{3}-F_{4}\right), \quad \bar{\iota}^{*} F_{5}=\lambda F_{5} .
\end{gathered}
$$

We will find $\overline{l_{6}}$-invariant surfaces $S_{\xi}$ in $\mathrm{CCI}_{\lambda}$. If $S_{\xi}$ is $\overline{l_{6}}$-invariant, then there exists a complex number $c \neq 0$ such that $c \sum_{i=1}^{4} s(\xi)_{i} F_{i}=\sum_{i=1}^{4} s(\xi)_{i} \bar{l}_{6}{ }^{*} F_{i}$. Then we have

$$
\left(\begin{array}{cccc}
-\lambda-c & -(\lambda-1) & 0 & 0 \\
0 & (\lambda-1)-c & 0 & 0 \\
-4 \lambda & -4(\lambda-1) & 1-c & \lambda \\
0 & 4(\lambda-1) & 0 & -\lambda-c
\end{array}\right)\left(\begin{array}{l}
s(\xi)_{1} \\
s(\xi)_{2} \\
s(\xi)_{3} \\
s(\xi)_{4}
\end{array}\right)=0 .
$$

From this equality, we know that either the equality

$$
\left(s(\xi)_{1}, s(\xi)_{2}, s(\xi)_{3}, s(\xi)_{4}\right)=\left(s(\xi)_{1}, 0,-\sqrt{-3} \lambda^{2}\left(4 s(\xi)_{1}-s(\xi)_{4}\right) / 3, s(\xi)_{4}\right)
$$


or the equality

(112) $\left(s(\xi)_{1}, s(\xi)_{2}, s(\xi)_{3}, s(\xi)_{4}\right)=\left(\sqrt{-3} \lambda^{2} s(\xi)_{2}, s(\xi)_{2}, 4 \lambda s(\xi)_{2} / 3,-4 \sqrt{-3} \lambda^{2} s(\xi)_{2} / 3\right)$

is satisfied.

Since $\left(s(\xi)_{1}, s(\xi)_{2}, s(\xi)_{3}, s(\xi)_{4}\right)$ satisfies the condition $L_{\lambda}{ }^{\mathrm{t}}\left(s(\xi)_{1}, s(\xi)_{2}, s(\xi)_{3}\right.$, $\left.s(\xi)_{4}\right)=0$, we have

$$
(-12 \lambda \xi-8(\lambda+1)) s(\xi)_{1}-4 \lambda s(\xi)_{2}+(2(\lambda+1) \xi+3) s(\xi)_{3}+\lambda \xi s(\xi)_{4}=0 .
$$

If (112) is satisfied, then the left-hand side is equal to $-16 \xi s(\xi)_{2} / \sqrt{-3}$ by the equality $\lambda^{3}=-1$. This is impossible since $\xi \neq 0$ and $s(\xi)_{2} \neq 0$. Hence, (112) is not satisfied.

We consider the case (111) next. By the condition $L_{\lambda}{ }^{\mathrm{t}}\left(s(\xi)_{1}, s(\xi)_{2}, s(\xi)_{3}, s(\xi)_{4}\right)=0$, it is easy to see that $\left(s(\xi)_{1}: s(\xi)_{2}: s(\xi)_{3}: s(\xi)_{4}\right)=\left(3: 0: 4 \sqrt{-3} \lambda^{2}: 24\right)$ and $\xi=\xi_{0}=\lambda-2$. Therefore, $S_{\xi_{0}}$ is $\overline{l_{6}}$-invariant and $S_{\xi_{1}}, S_{\xi_{2}}$ and $S_{\xi_{3}}$ are not $\overline{l_{6}}$-invariant.

By Lemma 4.6, if $S_{\xi_{0}}$ is isomorphic to $S_{\xi_{i}}$, then there exists the automorphism $\bar{\Phi}$ of $\boldsymbol{P}_{E}$ which commutes with an automorphism $\varphi$ of $E$ leaving $o$ fixed such that $\bar{\Phi}\left(S_{\xi_{i}}\right)=S_{\xi_{0}}$. However, $\bar{\Phi}\left(S_{\xi_{0}}\right)=S_{\xi_{0}}$ since $\bar{\Phi}=\bar{\iota}_{6}^{i}$ for some $i$. Thus, $S_{\xi_{0}}$ is not isomorphic to every $S_{\xi_{i}}(i=1,2,3)$.

We assume that $s\left(\xi_{i}\right)_{1}=1$ for $i=1,2,3$. Since $F_{\xi_{1}} \neq c \bar{c}_{6}^{*} F_{\xi_{1}}$ for every $c \in C^{*}$, we can assume that $\bar{\iota}_{6}^{*} F_{\xi_{1}}=c F_{\xi_{2}}$ for $c \in \boldsymbol{C}^{*}$. Since $F_{\xi_{2}} \neq d \bar{\iota}_{6}^{*} F_{\xi_{2}}$ for every $d \in \boldsymbol{C}, \bar{\iota}_{6}^{*} F_{\xi_{2}}$ coincides with $d F_{\xi_{1}}$ or $d F_{\xi_{3}}$ for $d \in C^{*}$. If we assume that $\bar{\iota}_{6}^{*} F_{\xi_{2}}=d F_{\xi_{1}}$, then we have $F_{\xi_{3}}=e{\overline{l_{6}}}^{*} F_{\xi_{3}}$ for $e \in C$. This contradicts that $F_{\xi_{3}}$ is not $\overline{\iota_{6}}$-invariant. Hence, we have $\bar{\iota}_{6}^{*} F_{\xi_{2}}=d F_{\xi_{3}}$ for $d \in C^{*}$. Thus, $S_{\xi_{1}}, S_{\xi_{2}}$ and $S_{\xi_{3}}$ are isomorphic to each other.

Acknowledgments. The author expresses his sincere gratitude to Professors Tadashi Ashikaga and Kazuhiro Konno for their valuable discussions and advice. He also thanks Professors Masanori Ishida, Shoetsu Ogata, Takeshi Kajiwara, Tomokuni Takahashi and Dr Mizuho Ishizaka for their encouragement and useful advice.

\section{REFERENCES}

[ 1 ] M. F. ATiYah, Vector bundles over an elliptic curve, Proc. London Math. Soc. (3) 7 (1957), 414-452.

[2] F. Catanese And C. Ciliberto, Surfaces with $p_{g}=q=1$, Problems inthe theory of surfaces and their classification (Cortona, 1988), 49-79, Sympos. Math., XXXII, Academic Press, London, 1991.

[ 3 ] F. Catanese and C. Ciliberto, Symmetric products of ellipticcurves and surfaces of general type with $p_{g}=q=1$, J. Algebraic Geom. 2 (1993), 389-411.

[4] R. Hartshorne, Algebraic Geometry, Graduate Texts Math. 52, Springer-Verlag, New York-Heidelberg, 1977.

[ 5 ] E. HoRIKawa, On algebraic surfaces with pencils of curves of genus 2, Complex analysis and algebraic geometry, 79-90, Iwanami Shoten, Tokyo, 1977.

[ 6 ] E. HoRiKawA, Algebraic surfaces of general type with small $c_{1}^{2}$, I, Ann. of Math. (2) 104 (1976), 357-388; Algebraic surfaces of general type with small $c_{1}{ }^{2}$, II, Invent. Math. 37 (1976), 121-155; Algebraic surfaces of general type with small $c_{1}^{2}$, III, Invent. Math. 47 (1978/79), 209-248; Algebraic surfaces of general type with small $c_{1}^{2}$, IV, Invent. Math. 50 (1979), 103-128; Algebraic surfaces of general type with small $c_{1}{ }^{2}$, V, J. Fac. Sci. Univ. Tokyo Sect. IA Math. 28 (1981), 745-755. 
[ 7 ] M. NambA, Geometry of projective algebraic curves, Monogr. Textbooks Pure Appl. Math. 88, Dekker, New York, 1984.

[ 8 ] T. ODA, Vector bundles on an elliptic curve, Nagoya Math. J. 43 (1971), 41-72.

[ 9 ] T. TAKAHASHI, Certain algebraic surfaces of general type withirregularity one and their canonical mappings, Tohoku Math. J. (2) 50 (1998), 261-290.

Mathematical Institute

TOHOKU UNIVERSITY

SENDAI 980-8578

JAPAN

E-mail address: sa0m04@math.tohoku.ac.jp 